41 | 2010

Le cheval : monture, nourriture et figure

\title{
Les parfaits coursiers du Naadam
}

The perfect racehorses of the Naadam

\section{Gaëlle Lacaze}

\section{(2) OpenEdition}

Journals

Édition électronique

URL : https://journals.openedition.org/emscat/1618

DOI : $10.4000 /$ emscat.1618

ISSN : 2101-0013

Éditeur

Centre d'Etudes Mongoles \& Sibériennes / École Pratique des Hautes Études

\section{Référence électronique}

Gaëlle Lacaze, «Les parfaits coursiers du Naadam », Études mongoles et sibériennes, centrasiatiques et tibétaines [En ligne], 41 | 2010, mis en ligne le 15 avril 2010, consulté le 13 juillet 2021. URL : http:// journals.openedition.org/emscat/1618; DOI : https://doi.org/10.4000/emscat.1618

Ce document a été généré automatiquement le 13 juillet 2021.

(c) Tous droits réservés 


\title{
Les parfaits coursiers du Naadam
}

\author{
The perfect racehorses of the Naadam
}

\author{
Gaëlle Lacaze
}

1 Organisée les 11 et 12 juillet, la fête nationale mongole porte le nom de Erijn gurvan naadam «Les trois jeux virils », couramment abrégée en Naadam ${ }^{1}$ « Le jeu ». La plus grande des festivités prend place à Ulaanbaatar, c'est la « fête nationale » (Ulsyn bajar), le «Naadam étatique» (Törijn Naadam). Des fêtes de moindre ampleur, également appelées Naadam, se tiennent aussi à l'échelle régionale à la même période. Les trois jeux qui sont au centre du Naadam sont la lutte, les courses de chevaux et le tir à l'arc. Tous trois sont des compétitions où des hommes s'opposent, mettant à l'épreuve leurs qualités viriles ${ }^{2}$. Aussi peut-on dire que le Naadam offre aux hommes l'occasion de défendre l'honneur de leur patrie (nutag) devant un vaste public.

2 C'est l'héritière d'une fête qui, jadis, avait lieu au printemps pour " ouvrir » l'été, se présentant comme un rituel de renouveau (Lacaze 1999-2000). Ne pas organiser un Naadam aurait constitué un présage de sécheresse pour l'année à venir et, pour autant que nos sources nous permettent de le savoir, il n'y aurait jamais eu d'année sans Naadam. De nos jours, le Naadam national est considéré comme « ouvrant » l'automne, préparant en quelque sorte la saison froide. Les Naadam organisés en même temps ou à sa suite dans les centres de provinces (ajmag) ou de canton (sum) visent aussi parfois à commémorer l'anniversaire de leur fondation ou un événement faste pour la région et ses habitants. Ceci permet à certains citadins de passer leurs vacances d'été à aller d'un Naadam local à un autre à travers tout le pays. À l'échelle nationale comme à l'échelle locale, le Naadam a une portée politique dans la mesure où il glorifie l'institution qui l'ordonnance. Diverses pièces de littérature orale font référence au Naadam comme à une institution tutélaire, ainsi qu'aux instances qui représentent le pouvoir politique ou religieux dont il célèbre la gloire. Tout Naadam sert, en outre, de support à des expressions identitaires.

3 Les trois « jeux » ne sont pas également présents dans ce type de fêtes. Ainsi le tir à l'arc semble y être de plus en plus rare. Le décret édicté par le gouvernement en 2003 pour en imposer la tenue dans tout Naadam est en fait inapplicable, faute de tireurs. Si l'utilisation des armes à feu, dès la fin $\mathrm{du} \mathrm{XIX}^{\mathrm{e}}$ siècle, a contribué à la désuétude du tir à 
l'arc, c'est l'extension de la pratique aux femmes, dans les années 1940, qui semble avoir entraîné sa disgrâce définitive (Lacaze 1999-2000, p.115). En revanche, le tir d'osselets qui avait acquis le statut de naadam en 1998, est depuis 2006 accueilli, lors du Naadam national, dans le stade d'Ulaanbaatar ${ }^{3}$.

Cet article décrit les courses de chevaux à travers les pratiques qu'elles suscitent, les croyances qu'elles véhiculent et la littérature populaire de tradition orale à laquelle elles donnent naissance. Les louanges et les autres glorifications destinées aux coursiers gagnants développent de nombreux aspects et représentations symboliques des courses de chevaux, allant de la description du choix du cheval en passant par celle des exercices d'entraînement. Nous examinerons, pour commencer, le déroulement des courses afin de mettre en évidence les qualités reconnues aux chevaux gagnants. Ensuite, nous décrirons les différentes techniques d'entraînement des coursiers : leurs « caractéristiques » initiales, les différentes étapes de leur préparation et les éléments de la course qui y sont consécutifs. Nous soulignerons, pour finir, comment les courses, les représentations symboliques qu'elles supportent et l'imaginaire auquel elles font référence caractérisent une idéologie propre aux courses et différente de celle des autres naadam que sont la lutte et le tir à l'arc ${ }^{4}$.

Naadam : public à cheval

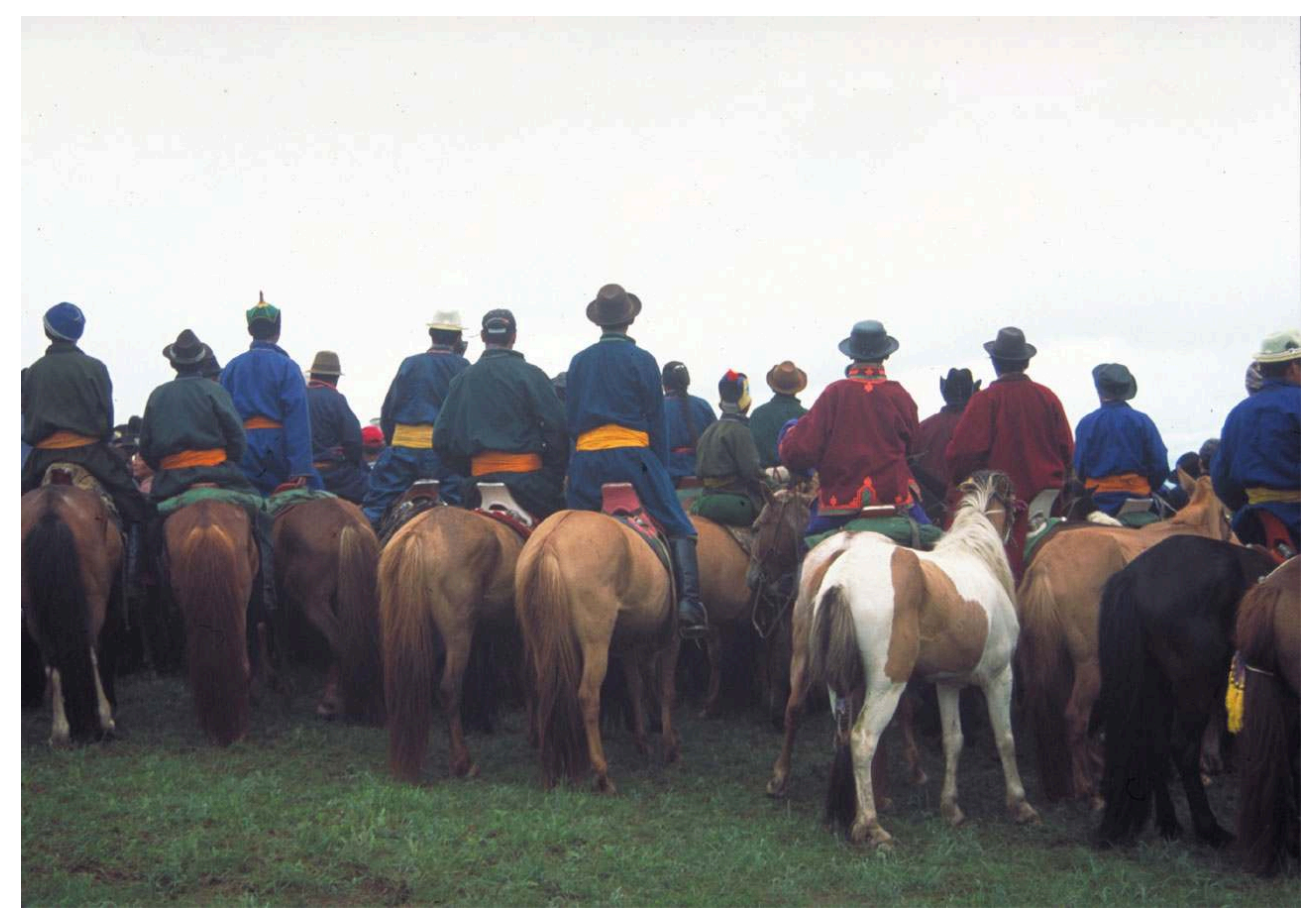

Naadam des 100000 chevaux, août 1998, Övörhangaj, Harhorin, jubilée de la capitale de l'ajmag et célébration du $700^{e}$ anniversaire de la ville

Photographie de Gaëlle Lacaze

\section{Les courses du Naadam}

5 En Mongolie, de nos jours, les hommes en général, et les citadins en particulier, s'intéressent davantage à la lutte qu'aux deux autres naadam, car ils sont plus à même de la comprendre et de l'apprécier. La lutte offre une expression virile sans lien direct 
avec l'économie d'élevage; elle ne souffre d'aucune connotation rurale et interpelle tout Mongol, cavalier ou non. Le tir à l'arc reste, aujourd'hui, le moins connu et le moins apprécié des naadam chez les Mongols.

Il semble que les courses de chevaux ont récemment été introduites dans le Naadam, même s'il reste difficile de savoir précisément quand elles y ont fait leur apparition ${ }^{5}$. Les sources accessibles consultées ne mentionnent que la lutte et le tir à l'arc pour les périodes qui précèdent la domination mandchoue en Mongolie orientale ${ }^{6}$. Et celle-ci a apporté des changements dans le Naadam ${ }^{7}$. Au cours du XviII siècle, l'organisation de Naadam se généralise dans d'autres rituels à l'ovoo « cairn "8.

7 Les courses sont, aujourd'hui, très appréciées des femmes et des éleveurs. L'emblème du Mongol est toujours lié au modèle du cavalier. Cependant, de nombreux citadins ne sont jamais montés à cheval. En outre, la population mongole s'inscrit actuellement dans un large mouvement de sédentarisation, tandis que le gouvernement adopte une politique de destruction du pastoralisme nomade.

Beaucoup de spectateurs et de participants viennent de toutes les régions du pays jusqu'à Ulaanbaatar afin d'assister au Naadam national des 11 et 12 juillet. Certains installent leur yourte principale, une petite yourte d'appoint (ilü̈ ger) ou une tente sur le lieu des courses de chevaux, à quarante kilomètres d'Ulaanbaatar'9. Un véritable campement s'y monte quelques jours avant le Naadam.

\section{L'organisation des courses}

9 Quelle que soit l'ampleur du Naadam, différentes courses sont organisées en fonction du statut reproductif et de l'âge des chevaux. Les hongres se répartissent en cinq catégories d'âge : des daaga " poulains de deux ans » aux ih nas, les "grands âges » - de six ans et plus - en passant par les šüdlen de trois ans, les hjazalan de quatre ans et les soëlon de cinq ans.

10 Dans les petits Naadam de province, les courses de chevaux constituent toujours l'animation de prédilection, on y trouve la plupart des catégories de coursiers. Tous les Naadam comportent une course d'étalons (azarga) et au moins trois courses de hongres, tandis que seul le grand Naadam de la capitale accueille la course des chevaux ambleurs (žoroo mor'). Celle-ci a lieu le 13 juillet, après la clôture des festivités officielles, lors du Naadam des entraîneurs (ujaačin naadam), qui ne comporte qu'une seule course. Les ambleurs y sont montés par les entraîneurs eux-mêmes et ne sont pas récompensés devant la tribune officielle. Aucune source écrite ne mentionne de louange aux ambleurs vainqueurs ni même le Naadam des entraîneurs, qui aurait été tardivement introduit dans les festivités nationales de la RPM.

11 Les daaga "poulains" et les žoroo "ambleurs » courent sur une distance de quinze kilomètres ; les šüdlen, vingt kilomètres ; les hjazalan, vingt-cinq kilomètres ; les étalons et les soëlon, vingt-huit kilomètres. La plus longue course, celle des ih nas, les chevaux de plus de six ans, fait trente kilomètres. Il n'y a pas de course en hiver, car les entraîneurs estiment qu'il est dangereux de faire courir un cheval en cette saison. Depuis peu, néanmoins, les premières courses de l'année ont lieu au cours des festivités du Nouvel $\mathrm{an}^{10}$, pendant Cagaan sar «le Mois blanc $»^{11}$. À cette période de l'année, le froid reste virulent et les animaux sont émaciés, car c'est la fin de l'hiver. Les courses du Cagaan sar, organisées principalement lors de compétitions sportives, notamment de 
lutte, ou pour les voyageurs et touristes hivernaux, ne comportent pas toutes les catégories de chevaux présentes durant les Naadam estivaux.

Concurrent du Naadam - avant la course

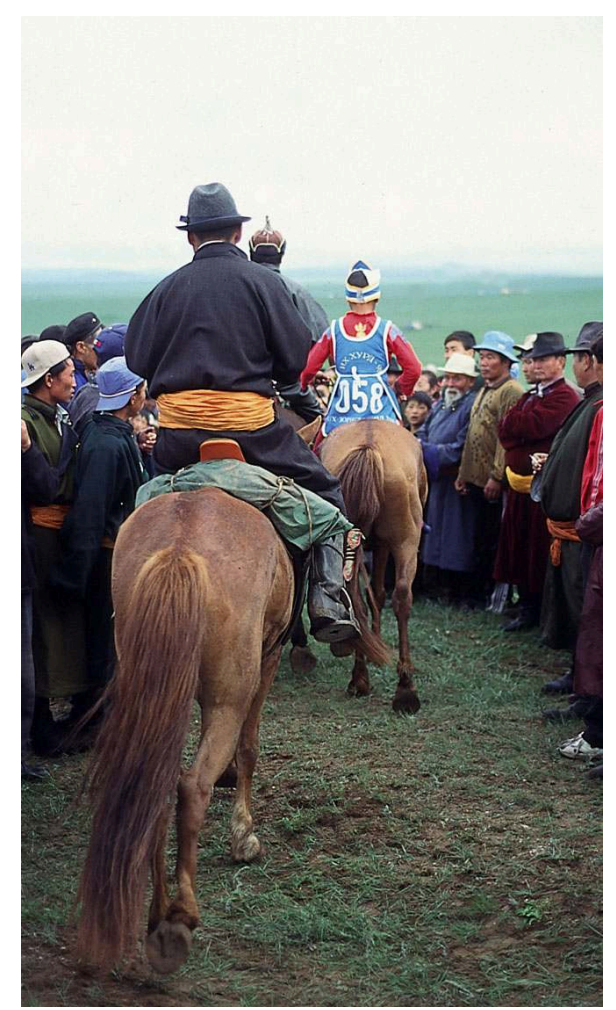

Naadam des 100000 chevaux, août 1998, Övörhangaj, Harhorin, jubilée de la capitale de l'ajmag et célébration du $700^{\circ}$ anniversaire de la ville

Photographie de Gaëlle Lacaze

12 Toutes les courses suivent des parcours spécifiques. Afin d'éviter les tricheries de la part de concurrents qui pourraient arriver en cours de route, nul ne connaît à l'avance le trajet emprunté par les participants, en dehors des membres de la commission chargée d'organiser les courses. Les coursiers franchissent une première barrière de contrôle, où l'examen de leurs dents permet de vérifier leur âge. C'est le point de départ initial officialisant la participation à la course. Ensuite, ils parcourent au pas, en se regroupant, le trajet inverse de la course, derrière une monture officielle dont le cavalier tient un étendard, généralement de couleur rouge ${ }^{12}$. Arrivant près du point de départ effectif, tous attendent son signal. Quand le coup de sifflet retentit, les chevaux font demi-tour et s'élancent au galop tandis que les cavaliers fendent l'air de leur cravache et entament la psalmodie du gijngo ${ }^{13}$. Tous rejoignent au grand galop l'arrivée, située au point de contrôle de l'âge. 
Concurrent du Naadam derrière le porte étendard, après le passage du contrôle

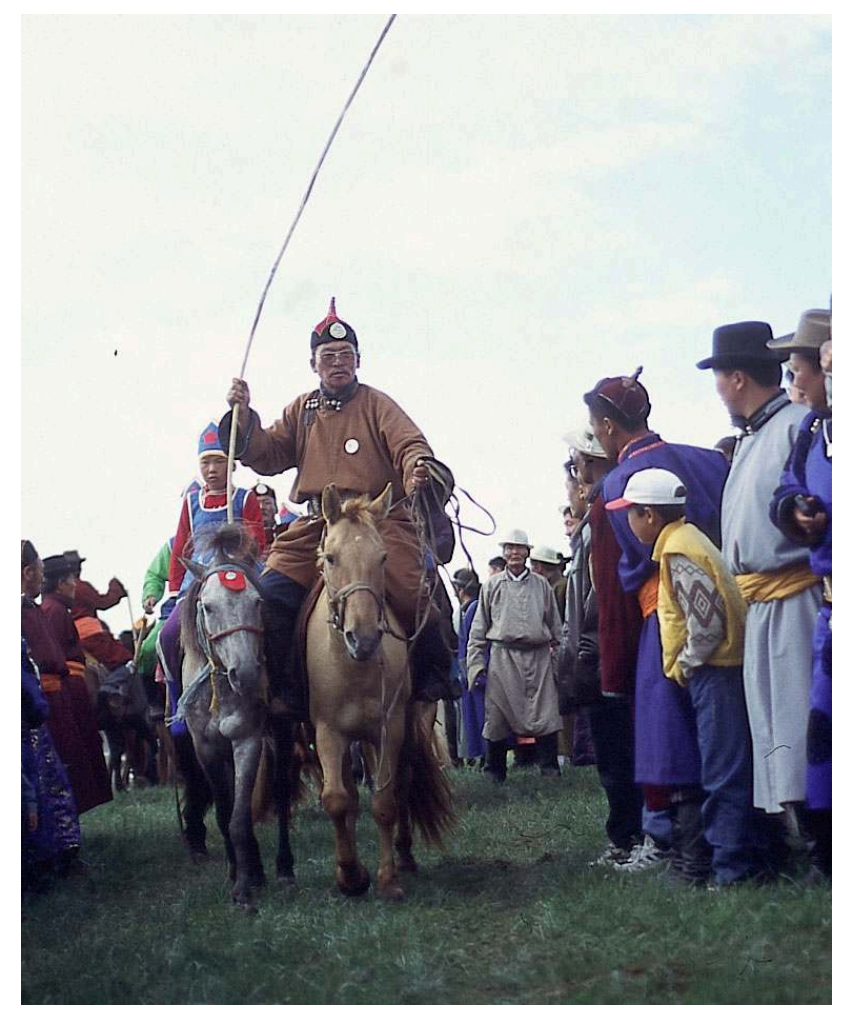

Naadam des 100000 chevaux, août 1998, Övörhangaj, Harhorin, jubilée de la capitale de l'ajmag et célébration du $700^{\mathrm{e}}$ anniversaire de la ville

Photographie de Gaëlle Lacaze

13 Au départ effectif de la course, le brusque demi-tour désarçonne souvent les cavaliers, des enfants de six à douze ans, garçons ou filles ${ }^{14}$. Un cheval sans cavalier se voit rétrogradé d'une place s'il franchit la ligne d'arrivée parmi les gagnants. Sont considérés comme gagnants les cinq premiers coursiers à franchir la ligne d'arrivée, appelés ajrgijn tav « les cinq de l'ajrag ", et leurs entraîneurs - ujaačin " ceux de la longe d'attache ». On honore également d'une louange $\left(\mathrm{col}^{15}\right)$ et d'une onction de lait de jument fermenté (ajrag) le dernier arrivé de la course des poulains daaga, à qui est attribué le nom de bajan hodood « riche estomac $»^{16}$. 


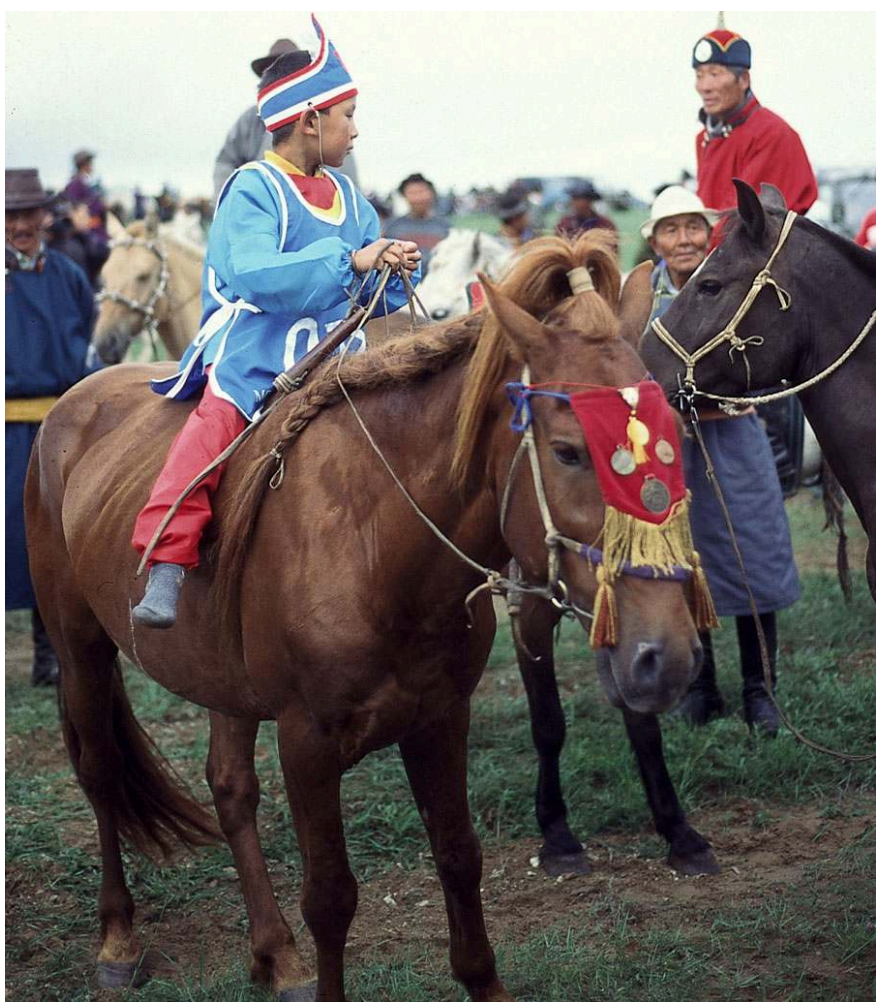

Naadam des 100000 chevaux, août 1998, Övörhangaj, Harhorin, jubilée de la capitale de l'ajmag et célébration du $700^{\mathrm{e}}$ anniversaire de la ville

Photographie de Gaëlle Lacaze

Parmi les cinq gagnants de chaque course, le premier, dit tümnij eh «premier de la multitude ", reçoit une médaille d'or, le deuxième et le troisième, une médaille d'argent, et le quatrième et le cinquième, une médaille de bronze. La remise des médailles s'accompagne d'une aspersion de lait de jument fermenté sur la croupe, l'encolure et le toupet du cheval. Cette aspersion constitue une récompense symbolique et explique le nom des gagnants, les ajrgijn tav « cinq du lait de jument fermenté ». Son évocation suffit à signifier la victoire dans les mor'ny col «louanges aux chevaux de course ", dont voici un exemple :

...Arrêté devant la tente

De la grande fête populaire du Naadam,

Je loue la gloire de sa fougue sauvage,

De cette illustre et si rapide monture,

Dont je tire les rênes de soie vers le haut

En ayant rempli à ras bord

Un calice à l'intérieur d'argent et l'embouchure d'or

D'un ajrag [battu] à l'aide d'un moussoir de cyprès et de santal.

Celui qui a gagné, monté au grand galop,

A augmenté encore sa vitesse

Il a couvert de joie

Et émerveille la foule

Il a réjoui son maître bienveillant.

L'honorable "premier de la multitude"

[Est] merveilleusement beau

Le fort et vigoureux "front de la fête"

[Est] une rapide monture à la grâce immense ! 


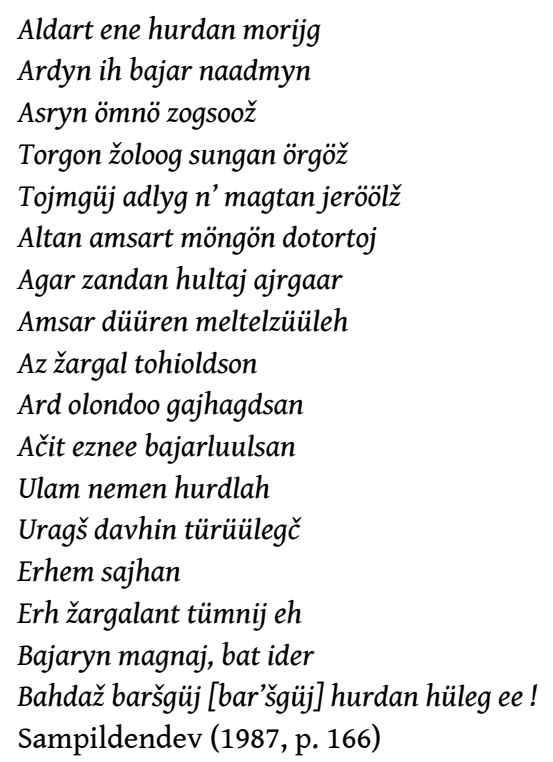

Le morio barigč « attrapeur de chevaux » chante les louanges du cheval gagnant et celles de son entraîneur avant d'effectuer l'aspersion d'ajrag devant la tribune officielle du Naadam. Son rôle ne se limite pas à cette démonstration prestigieuse car, auparavant, il a attrapé le coursier gagnant à l'arrivée - d'où son nom. Aujourd'hui, chaque attrapeur de chevaux dispose de témoins numérotés qu'il donne aux cavaliers en fonction de leur ordre d'arrivée ${ }^{17}$. Les morio barigč devant ainsi courir après les premiers, l'arrivée des courses provoque différents mouvements et entraîne un certain brouhaha dans le public, surtout du côté des éleveurs montés qui assistent aux courses sans mettre pied à terre.

Arrivée des courses : remise de témoin par les « preneurs »

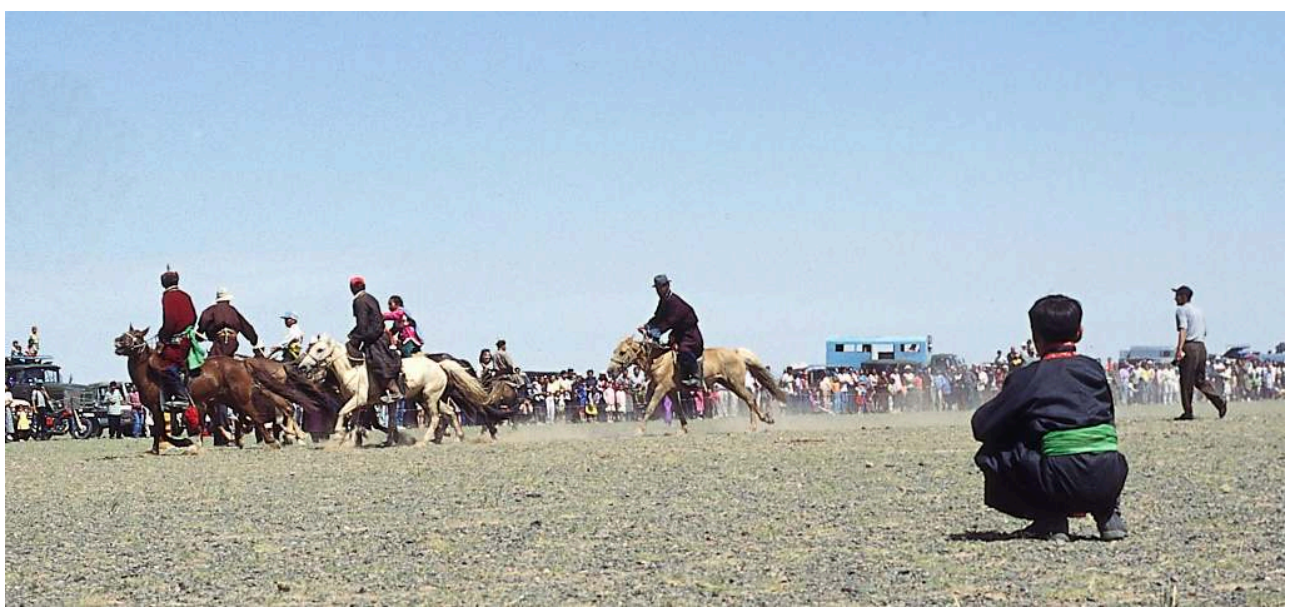

Naadam de sum, juillet 1998, Ömnögov', Bulgan

Photographie de Gaëlle Lacaze

\section{Les différents éloges du cheval}

Les louanges du cheval (mor'ny col) glorifient les coursiers gagnants en fonction de leur place d'arrivée. Le premier, appelé bajaryn magnaj «front de la fête» ou tümnij eh "premier de la multitude», le second, le troisième ainsi que le bajan hodood «riche 
estomac " possèdent leurs propres louanges. Dans ce genre littéraire, on trouve aussi des épithètes ${ }^{18}$ (čimeg), attribuées aux chevaux qui arrivent plusieurs années à la même place gagnante. Le cheval qui finit en tête d'une course pour la deuxième fois reçoit le titre de « monture qui augmente sa vitesse en courant [comme si] elle volait $»^{19}$. Pour la troisième année consécutive, il devient «monture bienheureuse du Hangaj fruitier " ${ }^{20}$; la quatrième année, "monture rapide que la tornade ne rattrape pas $»^{21}$; la cinquième, " monture [semblable à l'] élan à la rapidité sans pareil »"2 ; et la sixième, "monture rare qui court en tête des hongres ${ }^{23}$. Ces épithètes utilisent pour louer le gagnant des images empruntées à l'environnement et au monde naturel.

La même source mentionne les épithètes attribuées au deuxième cheval. Pour la deuxième victoire consécutive, on donne au second des courses l'épithète de " comète rouge de l'État $»^{24}$ et pour la troisième année, celle de "monture indestructible d'un bon État $»^{25}$. La quatrième, il reçoit l'épithète de «monture [qui fait] l'espoir de l'État souverain $»^{26}$, la cinquième, celle de «monture [qui fait la] fierté de l'État stable $»^{27}$ et la sixième, celle de "monture rapide de l'État révolutionnaire ${ }^{28}$. Les épithètes du deuxième cheval s'inscrivent dans un registre différent de celles attribuées au premier. Leur lexique emprunte ses références au monde réel et leurs modèles sont plus nationalistes, probablement parce que les épithètes adressées au second sont encore plus récentes que celles du premier. On trouve également, dans une louange au « riche estomac $»^{29}$, la glorification de la RPM en tant que destinataire des jeux :

Dans le grand festival du Naadam

Célébrant la ... année

De la très renommée

République de Mongolie

Indépendante et souveraine...

Bügd najramdah

Büren erht

Tusgaar togtnoson

Aldart mongol ulsyn...

Žilijn ojn...

Sampildendev (1987, pp. 173-174)

Les courses font l'objet de divers enjeux identitaires. Chaque joueur représente sa région d'origine, dont il prouve l'excellent équilibre naturel et social ${ }^{30}$. Pour le cheval de course, la mention de sa région renvoie en même temps à la provenance de l'animal, au rattachement administratif ou à la localisation du troupeau auquel il appartient et à la provenance géographique de son entraîneur. Ainsi, dans une louange au cheval arrivé le premier ${ }^{31}$, la première strophe présente le gagnant comme «le descendant d'un étalon de bonne race du troupeau numéro... de la coopérative... de l'ajmag... de la RPM souveraine! $»^{32}$. Une autre louange, dédiée au "riche estomac», glorifie les qualités du " perdant des perdants » en soulignant l'excellence de son entraînement et de son pedigree.

Il a été choisi parmi les hongres

Dans ce grand Naadam,

Il a été attrapé dans le troupeau

De la coopérative rurale... de la commune... de la province...

Il a galopé dans toutes les fêtes

Il a gagné tous les Naadam

Il est arrivé le premier parmi des milliers de hongres

En tirant sur les rênes de soie pour arriver

Il est arrivé le premier de la multitude de hongres 
En tirant sur les anneaux [de mors] en fer

On le dit beau en suivant ses ancêtres

On le dit rapide en accord avec son entraînement...

Ih bajar naadamd

... ajmgijn ... sumyn ... negdlijn

Aduunaas n' bar'san

Agtnaas n' šilsen

Najr bühend davhihaar

Naadam bühend türüüleheer

Mjangan agtny türüünd ireheer

Mjandsan žoloo sungan ireheer

Tümen agtny türüüd ireheer

Tömör zuuzaj sungan ireheer

Udmaa dagaad sajhan gedeg

Ujaa n' tohirood hurdan gedeg...

Bajan hodoodny col (Sampildendev 1987 pp. 173-175)

Différents rituels placent les Naadam sous la protection d'esprits protecteurs ou de divinités. La cérémonie d'ouverture du Naadam de 2007 comportait ainsi une danse de guerriers gengiskhanides, une danse chamanique et un rituel du $\mathrm{Cam}^{33}$. Parmi les sources écrites consultées, rares sont celles qui mentionnent le pouvoir régulier bouddhique. L'ouverture d'un mor'ny col $^{34}$ recueilli dans les années 1960 chez des Mongols Üzümčin (ajmag de Dornod, sum de Bajantümen, baga de l'Amiral Bajasgalan), population orientale installée de part et d'autre de la frontière sino-mongole, évoque néanmoins la protection bouddhique.

En ce jour rempli de quiétude,

Nos moines bouddhistes ayant répandu leurs grâces en priant rituellement

Et en faisant une offrande à l'ovoo du Mont Sumeru ${ }^{35}$, à l'esprit tutélaire de l'eau et

des montagnes...

Amgalan hotol tögsgösön ene sajn ödör

Avralyn ornoo orojn čimeg lamtnaa urin zalaad

Aguul usny ezen han Sümber ovoo juugaa tahiulaad...

Sampildendev (1987, pp. 153-154)

\section{Les gagnants}

Dès que les coursiers s'annoncent au loin, signalés par un nuage de poussière et un vrombissement sourd, le public s'agglutine autour de la ligne d'arrivée. Les louanges du Naadam développent différentes métaphores décrivant les perceptions, essentiellement auditives et visuelles, provoquées par l'arrivée. La vitesse du gagnant est évoquée, entre autres, par la métaphore du vent, de la tornade, du tonnerre ou de l'éclair. La fulgurance de sa course est suggérée par l'intensité du son qu'elle dégage et la poussière qui l'annonce. L'un des mor'ny col précédemment cités réunit plusieurs des images classiques de ce genre littéraire. Après l'ouverture mentionnant la purification des moines bouddhistes, la louange du cheval gagnant énumère ses qualités en évoquant les sensations provoquées par la vitesse de sa course et la puissance de son galop.

La louange énumère de la manière suivante les multiples qualités

Qui distinguent ce poulain d'un an des autres merveilleuses montures

Qu'il affronte au sein du troupeau innombrable

Son arrivée siffle comme le vent

Sa poussière pleut comme une tornade 
Ses sabots résonnent comme la grêle

Ses traces sont semblables [à celles] du dragon...

Tümen üržilt buman toot sürgijn unagaa

Sü̈̈geež/Süjgež ireh n' salhi met

Suvrah toos n' egüül met

Tuurajn tusgal n' möndör met

Tunalah daguu n' luu met

Tusgal ihtej ad'naan hülgijn egüün dor

Togtoon daguulah col n' juu ve gevel

(ibid., p. 154)

21 Une autre strophe de ce texte reprend la description sensorielle de la course du gagnant :

On récompense affectueusement

De l'ensemble des cadeaux suivants ...

Le cheval ... au museau chaud

Qui vole comme une flèche,

Qui est comme l'éclair dans la nuit noire,

Comme l'écho des falaises rocailleuses

Et comme un mirage des steppes du Hangaj !

Harvasan sumny süjgee met

Haranhuj šönijn cahilgaan met

Had čuluuny dujraa met

Hangaj talyn zereglee met

... Haluun hošuutaj mor'

Hamt ... (tödön) belgeer

Hajrlan šagnav aa!

(ibid.)

22 Une autre louange évoque la vitesse de la course du gagnant en des termes proches, mais en utilisant des images plus concrètes :

Avec son courage infaillible,

Sa tête de topaze

Et ses yeux noirs [comme] les fruits

du merisier

Il surgit en galopant de très loin,

Il surgit de derrière les montagnes

Il galope en jouant avec sa queue

Et s'effarouche de son ombre

Il galope en jouant avec sa tête

Et s'effarouche de sa poussière

Il court à la surface de la terre

Et tire sur ses anneaux [de mors]

Il galope en agitant son mors

Et réduit [la distance entre] les terres éloignées

Mojl har nüdtej

Molor erdene tolgojtoj

Mohošgüj eremgij čadaltaj

Hazaar hajalan davhidag

Havijn gasryn tovčlon davhidag

Zuuzajgaaraa zudran güjdeg

Zuuryn gazryn točloon güjdeg

Tolgojgooroo toglon davhidag

Toosnoosoo busgan davhidag

Süüleeree toglon davhidag

Süüderneesee busgan davhidag 
Uulyn caanaas undran davhidag

Ulam hojnoos degden davhidag

(ibid., pp. 165-166)

On qualifie les gagnants à répétition de l'épithète bariand güjceggdešgüj, bacdam hüleg "fière monture, qui n'est pas rejointe à l'arrivée " ${ }^{36}$ (Sampildendev 1987, p. 173). Parallèlement, plusieurs louanges illustrent la vitesse du gagnant en évoquant la peine qu'éprouve l'attrapeur de chevaux à saisir le coursier :
Arrivée en tête de la course
Du grand Naadam national,
Avec sa rapidité étonnante
Et la vitesse de l'éclair,
Cette monture si rapide
A creusé la surface de la terre
Elle est parvenue à faire voler
l'enfant qui paraît si jeune
Et elle a réussi à dépasser
le médiocre preneur... ${ }^{37}$
Önör hurdan ene hüleg
Baragtaj barigčijg dajrčihmaar
Baga šig hüühdijg hijsgečihmeer
Gazryn hörsijg ongilson
Gavšgaj hurdan gišgelttej
Gajhamšigt hurdan čadlaaraa
Ulsyn ih naadmyn
Uraldaany türüünd irehdee!
(ibid., p. 163)

24 Certaines louanges glorifient les gagnants comme symboles de la grandeur des courses et du Naadam au sein duquel elles sont organisées. Une louange du « riche estomac $»^{38}$ présente le grand perdant comme un emblème fédérateur des sentiments identitaires liés au Naadam, c'est-à-dire, entre autres, la «mongolité », le nationalisme et l'identité halh ${ }^{39}$.

Le bonheur fédérateur de cette fête [est]

Le symbole de bienvenue de la fête à venir

Celui qui doit se reposer de sa fatigue [possède] la légèreté d'une tête de flèche

Celui qui arrive en courant sans cravache [possède] les caractéristiques de la

monture

Celui qui arrive en bondissant [est] un cerf du Hangaj

Ce cheval, emblème populaire,

Est le symbole d'un bien être stable

Ene najryn üdeh bajar

Ireh najryn ugtamžijn bileg

Tav'saar zütgegč höngön zev

Tašuurgüj güjgeed iregč hülgijn šinž

Dü̈lleed iregč hangajn buga

Tümnij togtool ene mor'

Bat lutan enh mendijn sajhan bileg

(ibid., p. 175)

Cette louange dit aussi : "on se souviendra longtemps avec joie de celui qui émerge d'entre les nombreux chevaux de la Nation en train de galoper avec l'étendard rouge... $»^{40}$. Ainsi et malgré sa défaite, le « riche estomac » reste un modèle d'excellence qui illustre la grandeur de la "Nation ». Il fait la fierté de son propriétaire, qui a su le choisir et l'entraîner, ce qui aurait dû assurer sa victoire. Le perdant des perdants est 
décrit comme parfait et son entraînement comme parfaitement adapté à ses qualités. Sa défaite est le plus souvent imputée à l'absence de chance, aux obstacles de la route voire à l'inexpérience du cavalier.

Les épithètes peuvent récompenser jusqu'aux vingt-cinq premiers arrivés, gratifiés en outre d'un certificat. On y trouve notamment «la monture intrépide qui réjouit son maitre » ou « la monture très rapide, bonheur du peuple » ${ }^{41}$. Une autre louange rappelle toutes les instances glorifiées par la victoire dans les courses du Naadam.

Parmi les montures précieuses,

Ce cheval a émerveillé le commun des mortels

Et redressé le front de son propriétaire,

Il a ravivé la notoriété du troupeau de ses semblables,

Et a réjoui les pensées de la foule,

Il est devenu l'ornement du Naadam universel,

Il a laissé derrière [lui] une traînée de poussière brûlante,

Il a laissé derrière [lui] des milliers de hongres...

Tümen agtyg ardaa orhiž

Töönörsön toosyg butargan orhiž Tü̈̈gemel naadmyn čimeg bolž

Tümen olny setgelijg bajasgaž

Ižil sürgijn aldryg manduulž

Ezen hünij magnajg tenijlgež

Engijn olond gajhamšig bolson

Erdenijn hüleg mor' egüün dor...

Mor'ny col (Sampildendev 1987, pp. 153-155)

Durant les Naadam, le cheval gagnant et son entraîneur sont officiellement récompensés devant la tribune accueillant les organisateurs des jeux. Le cavalier n'est pas explicitement convié à ces récompenses publiques et officielles. S'il y prend part, c'est au titre du couple qu'il forme avec la monture récompensée. Les louanges destinées aux chevaux gagnants évoquent rarement le cavalier, car il ne joue qu'un rôle mineur dans la course comme dans la victoire. Ses erreurs sont néanmoins invoquées pour expliquer la défaite du « riche estomac». 


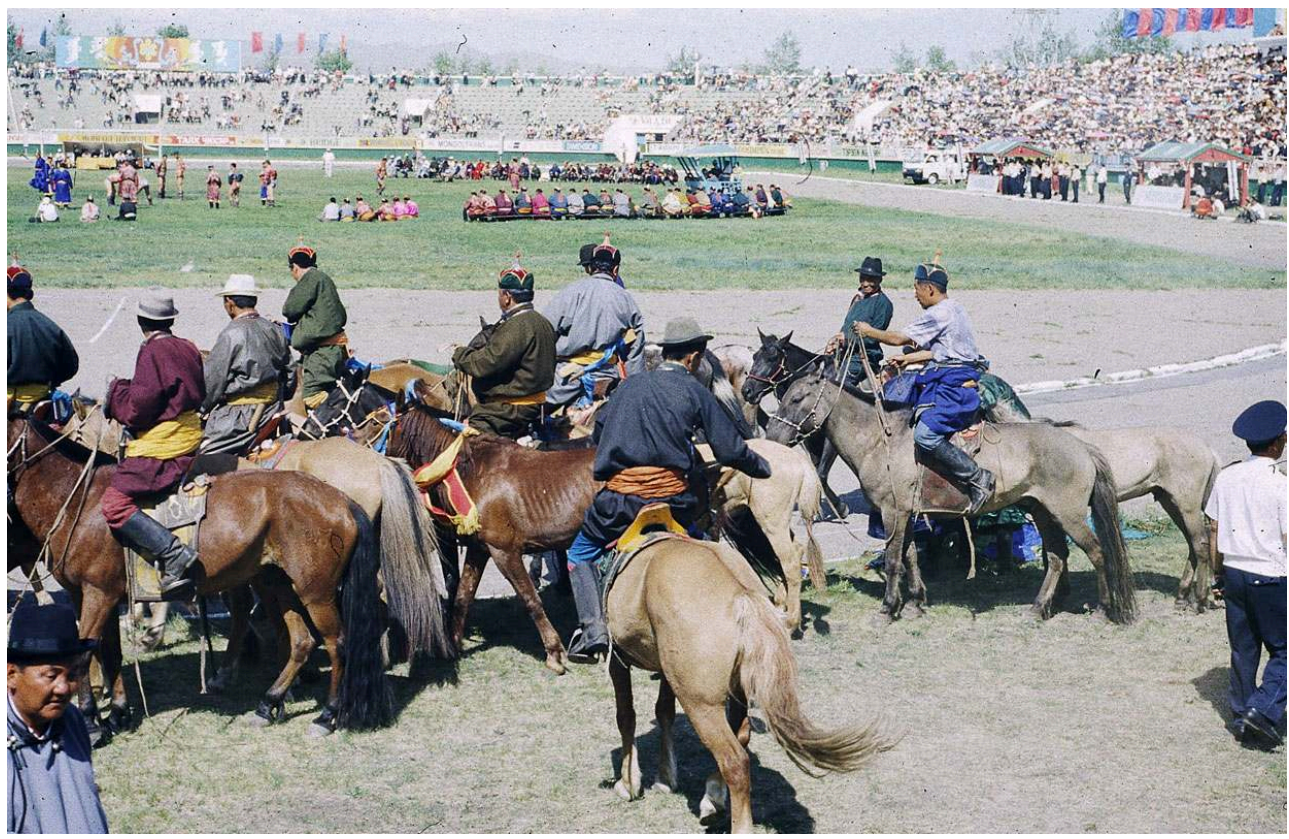

Stade d'Ulaanbaatar, juillet 1993

Photographie de Gaëlle Lacaze

Hormis l'éloge de la course du gagnant en des termes justifiant sa victoire, les louanges développent plusieurs motifs poétiques soulignant la qualité de son entraînement. Examinons maintenant en quels termes la poésie orale du Naadam récompense symboliquement l'entraîneur des chevaux de course, appelé ujaačin «celui de l'attache $»^{42}$.

\section{La préparation}

Beaucoup d'éléments importent dans la préparation des coursiers du Naadam : leur choix, leur entraînement, les soins accordés à leur harnachement et leurs parures. Le choix du coursier dépend du savoir-faire des entrâneurs, de leur connaissance des " caractéristiques du cheval» (mor'ny šinž) ${ }^{43}$. Les louanges évoquent également les soins apportés au costume de course du cheval (coiffure des crins et harnachement) et à celui de son cavalier.

\section{Le choix du coursier}

Les motifs des « louanges du cheval » (mor'ny col) reprennent les « caractéristiques » du " cheval rapide " (hurdan mor'), celui qui doit être choisi pour participer aux courses du Naadam. Ces « caractéristiques » concernent sa physionomie : la forme des sabots, des jarrets, de la croupe, des cuisses, des antérieurs et des postérieurs, mais aussi de la mâchoire et des oreilles ou encore l'éclat des yeux. Les métaphores les plus courantes utilisent le registre animal pour signifier la puissance et le registre floral ou minéral pour décrire la beauté du cheval rapide.

31 Le cheval rapide possède une encolure fine et une grosse tête. Sa croupe doit être inclinée et large afin de garantir l'écartement des postérieurs. Les flancs bombés et les 
jambes écartées assurent une bonne tenue au sol. Ils caractérisent les animaux endurants et vigoureux. Les louanges destinées au gagnant le comparent à l'éléphant ou au lion blancs. Elles lui attribuent la puissance du tigre. Elles glorifient sa vitesse, son endurance et sa force. Elles comparent la vitesse de sa course à celles du vent ou du loup. Ses sabots, larges et arrondis, sont régulièrement assimilés à un dais, à la roue des transmigrations du bouddhisme ou à un calice.

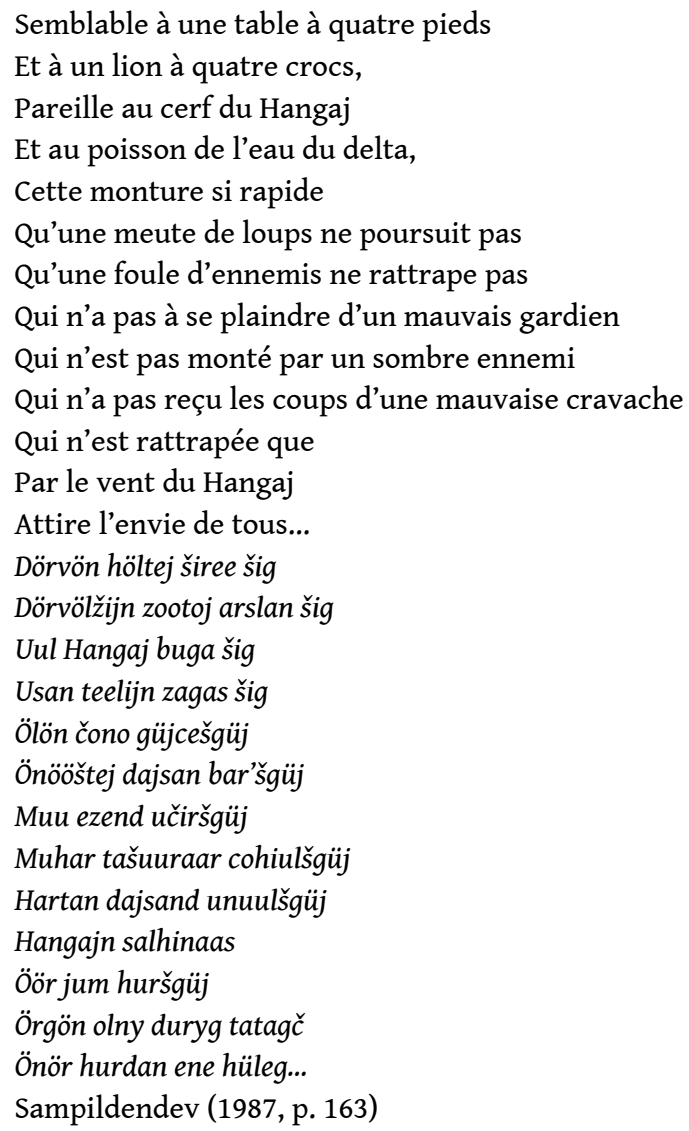

Les louanges vantent la forme de ses yeux, celle de ses oreilles et de sa langue, qui indiquent l'état de ses organes internes, respectivement son cœur, ses reins et son foie. Certaines métaphores visent à mettre en évidence des qualités indispensables aux coursiers. Ainsi, l'emphase portant sur les naseaux - qui doivent être larges et bien arrondis - et le poitrail - donc la cage thoracique et les poumons - soulignent les grandes capacités respiratoires du «cheval rapide». Le souffle constitue d'ailleurs le motif de nombreux vers de la littérature orale dédiée aux courses. Ces qualités externes renvoient à des qualités internes, selon des correspondances généralement gardées secrètes par les éleveurs. Choisi pour des " caractéristiques » signalant sa rapidité, le cheval gagnant remplit son rôle en remportant la victoire. Les mor'ny col reprennent donc les éléments physiologiques pour lesquels l'entraîneur l'a sélectionné et entraîné. Un extrait d'une louange répandue parmi les Mongols occidentaux illustre tous ces éléments ${ }^{44}$.

Si on mentionne les signes de hardiesse

De cette rapide monture,

Qui est arrivée en premier

Du troupeau innombrable,

S'envolant tel un faucon

En montrant des tendons à la parfaite rapidité : 
Elle possède au complet les signes du cheval

Rapide comme la biche maral,

Elle possède quatre canines [semblables aux défenses] de l'éléphant

Six vertèbres harmonieuses,

Quatre sabots sculptés,

Deux oreilles [en forme] de magnolia,

Et de belles dents [à l'éclat] de perle,

Elle possède une large et longue croupe révélée

Par l'attache et les « sauts » üserge ${ }^{45}$,

Dans son corps se retrouvent au complet

Les meilleures caractéristiques des chevaux :

Un corps solide et bien construit,

Une belle queue grisâtre ${ }^{46}$,

Une belle et soyeuse crinière

Et les beaux reins du lièvre.

Tüg tümen agtny dundaas

Tögs hurdan šandsaa üzüülž

Harcaga šuvuu šig nisež

Hamgijn türüünd iregč

Ene hurdan hölgijn

eremgij šinžijg durdval :

Zaan dörvön sojootoj

Zaamal zurgaan hüzüütej

Zasmal dörvön tuurajtaj

Zambaga hojor čihtej

Suvd sajhan šüdtej

Sogoo šig hurdan

Sunagar örgön tašaataj

Sojlgo ujaa n' tohirson

Sonž šinž n' bürdsen

Tuulaj sajhan zootoj

Tunamal sajhan deltej

Tunsag [sic] sajhan süültej

Tuhlag badiruun bijetej

Moriny sajn šinžijg

Mön bijedee bürdüülsen.

Une autre louange aux chevaux gagnants (Türü̈lsen mor'ny col) use de métaphores dont les références sont empruntées aux croyances et pratiques bouddhiques: la roue qui représente le cycle des transmigrations, le lien sans fin ou «lien du bonheur » (ölzij), dessiné un peu partout à des fins propitiatoires, la conque utilisée comme trompe, le lotus sur lequel repose le Bouddha, l'océan mythique ...

Je chante pour attribuer un titre

Qui qualifie la merveilleuse monture aux multiples qualités

Ce bon cheval a des sabots [ronds comme la roue] du cycle des transmigrations.

Il a la queue [en forme] d'un beau dais ${ }^{47}$,

La poitrine nouée d'un « lien du bonheur »,

Les dents [en forme] d'une conque blanche,

La tête [en forme] d'une belle cruche,

Les oreilles [en forme] du lotus,

Les yeux [en forme] de poisson de l'océan

Et la crinière [en forme] de crêtes lunaires.

Saran jührijn deltej

Sam'dyn zagasan nüdtej

Sam'jaa badmyn čihtej

Sajhan homhyn tolgojtoj 
Cagaan lavajn šüdtej

Zangidmal utsan ceežtej

Sajhan duasyn süültej

Sansryn hürden tuurajtaj

Sajn mor' ad'naan hüleg mön hemeen

Colyg togtoon duudna aa!

(ibid., p. 154)

\section{L'entraînement}

La préparation du Naadam national commence un mois environ avant les festivités. Elle concerne essentiellement le cheval, le cavalier n'ayant qu'un rôle mineur. Il s'entraîne de manière informelle, seul et sans ritualisation. Il doit surtout faire preuve de son aisance à cheval. Chaque entraîneur, généralement le père de l'enfant, possède des techniques pour préparer le cavalier, mais celles-ci ne font pas l'objet d'un savoir-faire prestigieux et valorisé. Les sources écrites disponibles sur le Naadam mentionnent rarement le cavalier et les éleveurs sont pour la plupart muets sur sa préparation.

L'entraînement débute en juin. Dans le centre du pays, la steppe se couvre de troupeaux ou de chevaux isolés qui la traversent au galop et rejoignent progressivement la capitale. Elle s'anime de rassemblements semi-festifs ${ }^{48}$ d'entraîneurs de chevaux de course. Certains prennent l'allure d'un véritable Naadam, attirant une foule de spectateurs. Les techniques d'entraînement des coursiers constituent une autre partie du savoir-faire de l'éleveur.

Un entraîneur attache un cheval à l'ujaa

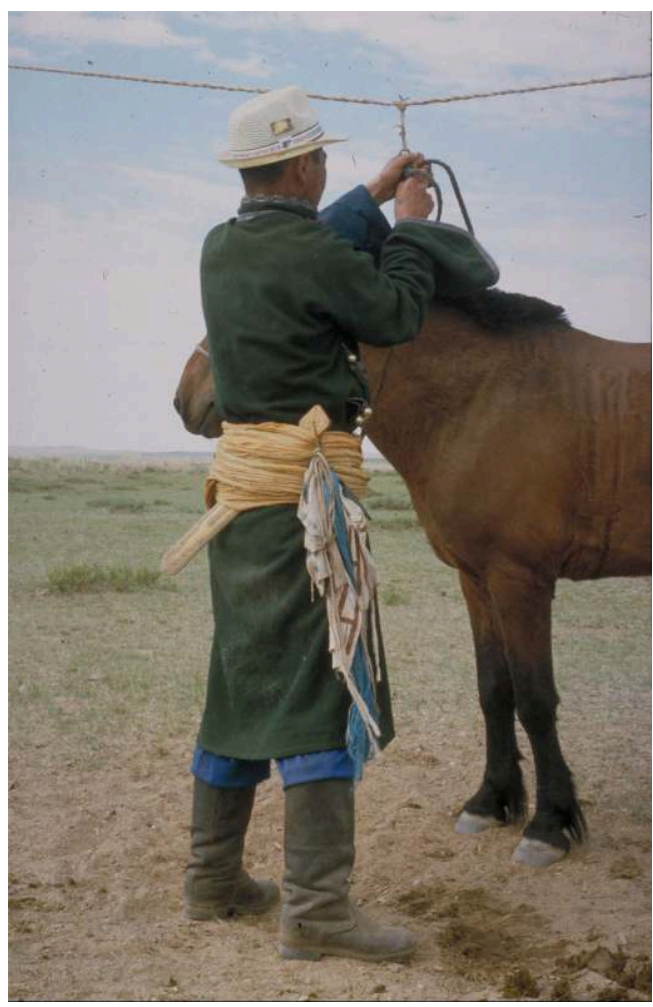

Juillet 1998, Gov'-Altaj, Tögrög

Photographie de Gaëlle Lacaze 
e verbe soj- signifie « attacher (un coursier pour l'entraîner aux courses) ». On emploie l'expression "longe et attache » (ujaa sojlgo) pour désigner l'entraînement aux courses hippiques du Naadam. Une grande partie de la préparation consiste, en effet, à attacher le coursier à la longe ujaa sous le soleil, parfois couvert d'une couverture de feutre, afin de le faire davantage transpirer. La bride du cheval rapide est nouée au plus court, de manière à ce que l'animal ne puisse pas brouter. L'attache diurne alterne avec l'alimentation nocturne du cheval, conduit chaque soir sur les sommets afin de se rassasier de l'herbe grasse des hauts pâturages. L'entraîneur organise quotidiennement de petites courses, les «sauts » (üserge), dont la distance est plus courte que celle des compétitions officielles pour chaque catégorie de coursier. Le savoir-faire de l'entraîneur réside dans une alternance adéquate entre l'attache de transpiration, les courses de préparation et la richesse de l'alimentation nocturne ${ }^{49}$.

\section{Préparation : üserge}

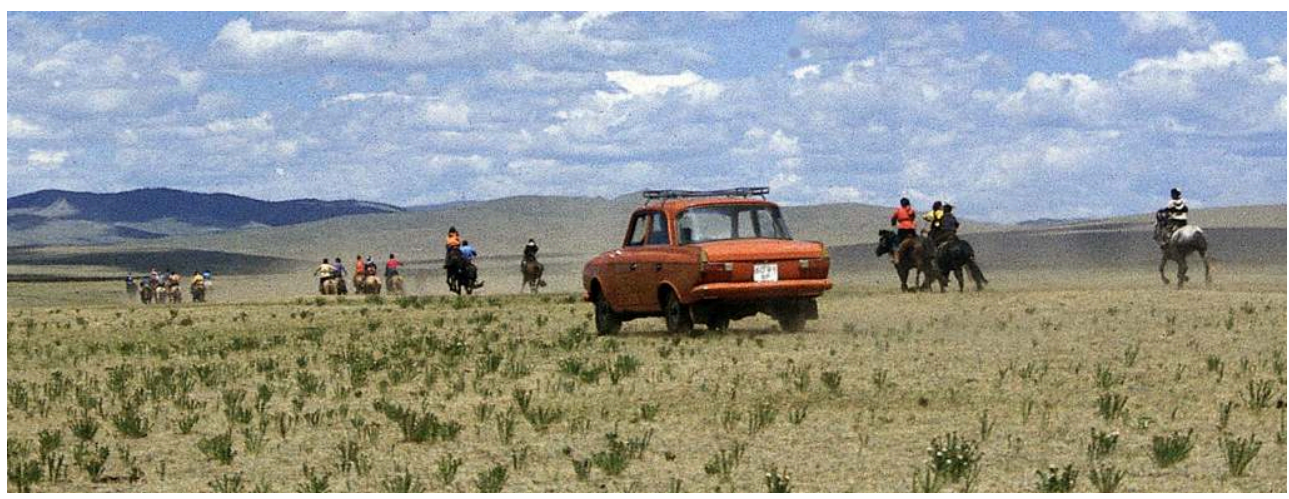

Juin 1998, Töv, Lun'

Photographie de Gaëlle Lacaze

Préparation : üserge

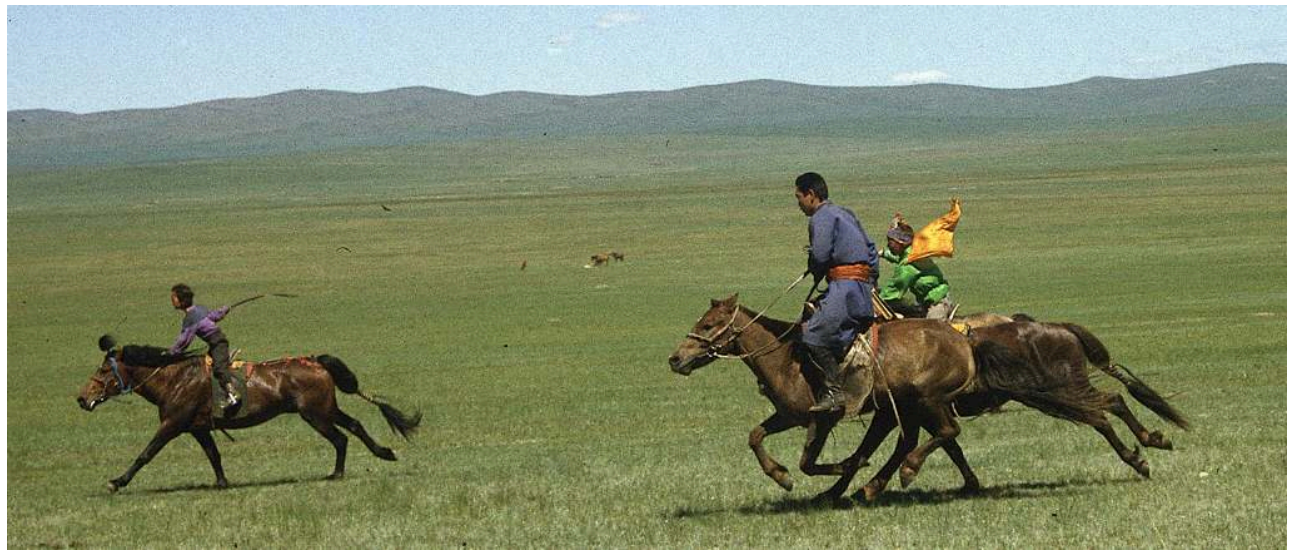

Juin 1998. Övörhangaj, Vallée d'Ongon

Photographie de Gaëlle Lacaze

La sueur du cheval gagnant possède un rôle propitiatoire. Au cours de l'entraînement, l'éleveur prend un peu de la sueur de chaque coursier sur un linge qu'il dépose ensuite sur son autel domestique, généralement situé en dessous des bâtons de sueur coincés sous les perches du toit, quand ils ne servent pas durant l'été. Il garantit ainsi la chance 
de ses chevaux. Pendant le Naadam, à l'arrivée des courses, les spectateurs se précipitent pour récupérer la sueur des gagnants sur un linge qu'ils conserveront ensuite sur l'autel familial afin de se protéger durant l'année, jusqu'au Naadam suivant ${ }^{50}$.

\section{Bâton de sueur}

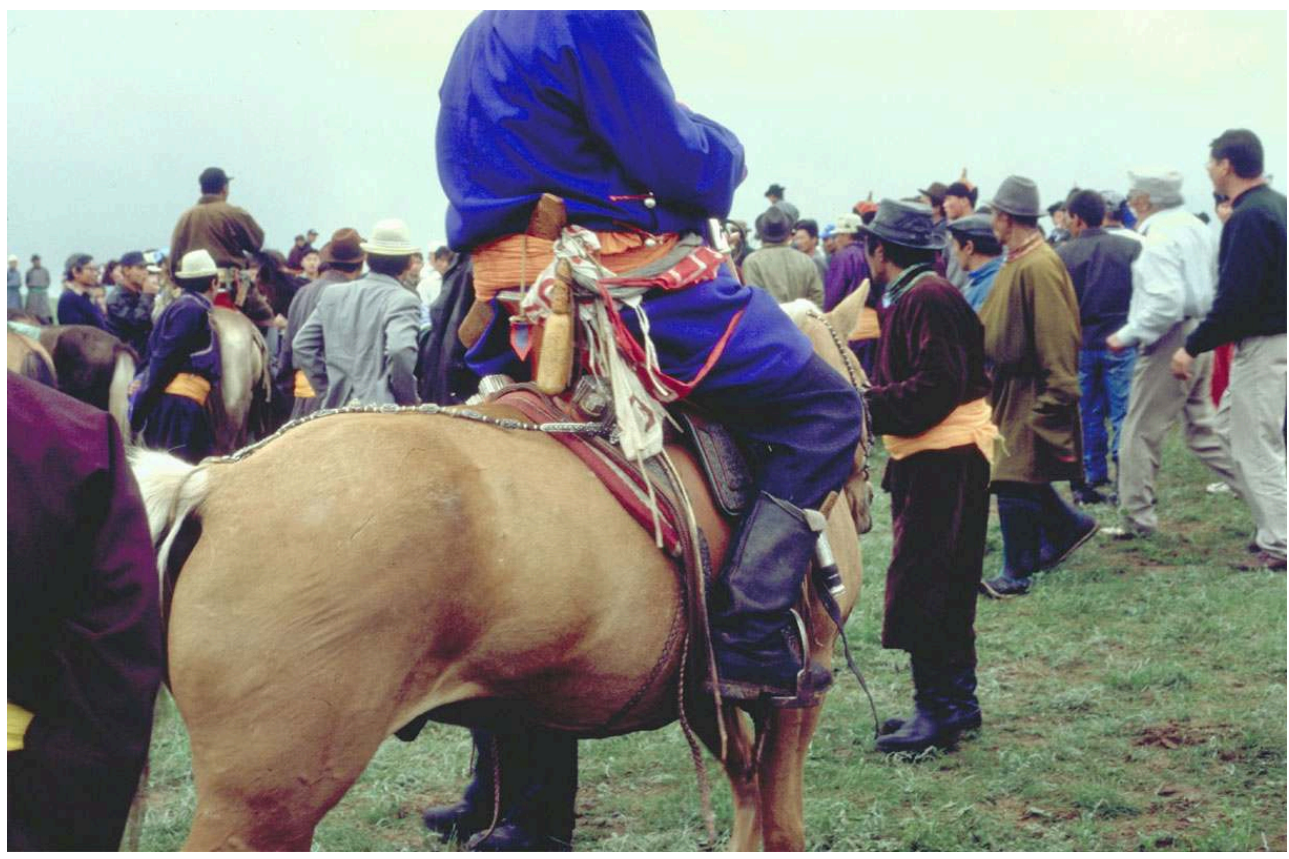

Naadam des 100000 chevaux, août 1998, Övörhangaj, Harhorin, jubilée de la capitale de l'ajmag et célébration du $700^{\mathrm{e}}$ anniversaire de la ville

Photographie de Gaëlle Lacaze

Rassemblement lors d'un üserge

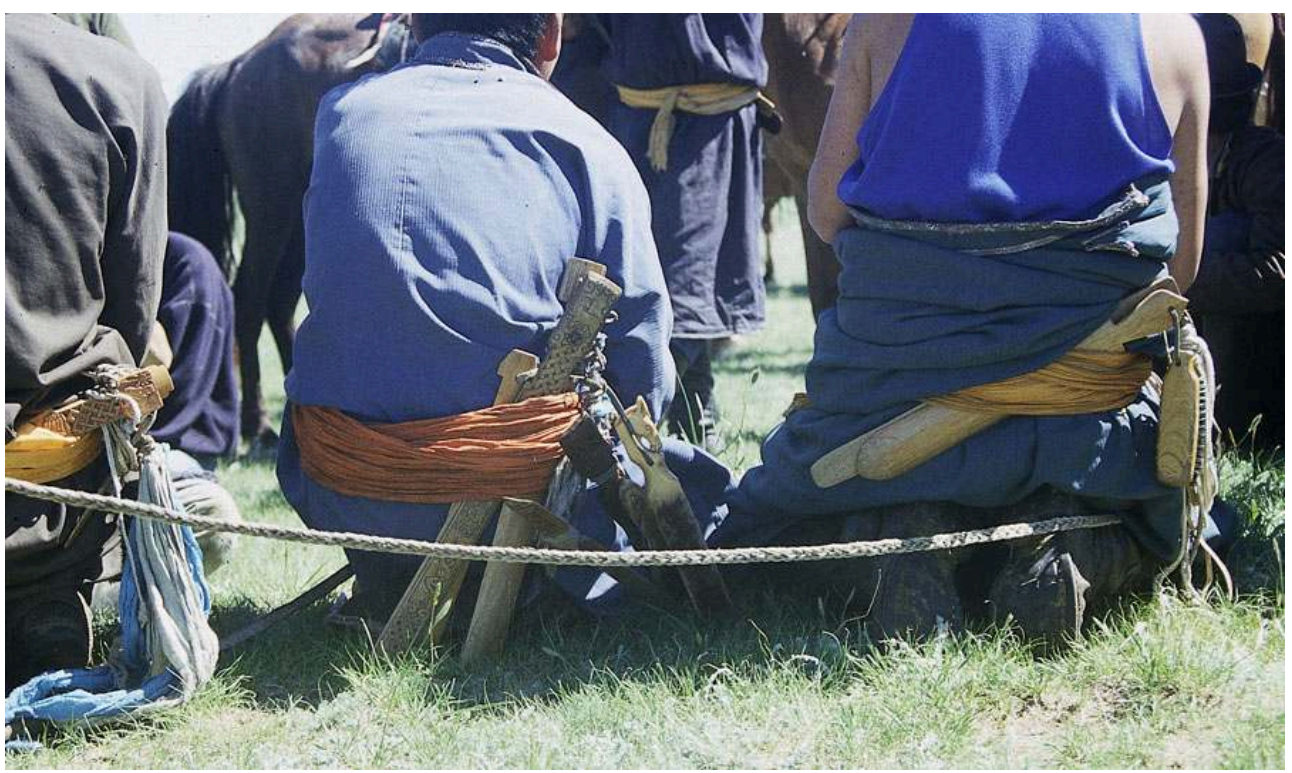

Gros plan : bâton de sueur. Juin 1998, Hentej, Bajan Ovoo

Photographie de Gaëlle Lacaze 


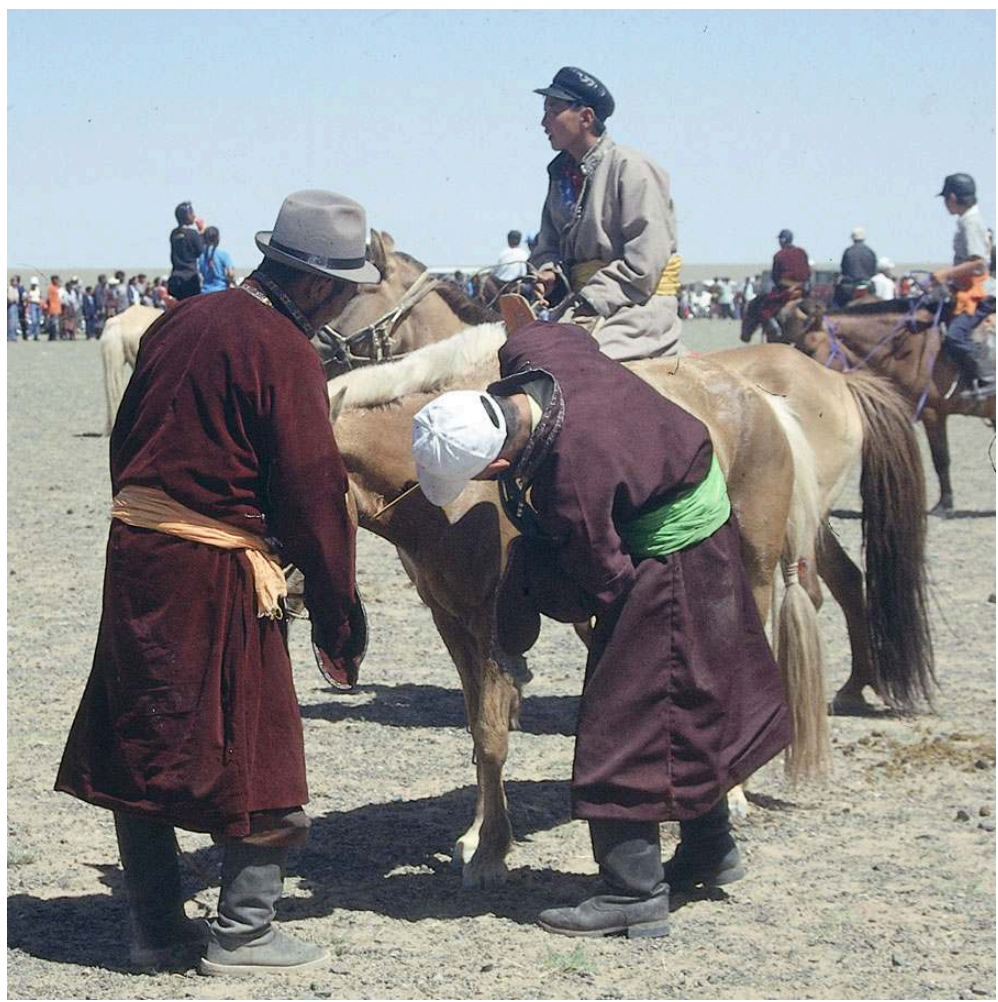

Naadam de sum, juillet 1998, Ömnögov', Bulgan

Photographie de Gaëlle Lacaze

Les « sauts » préparent le cheval à courir sur de longues distances. Ils ne constituent pas un thème répandu des louanges glorifiant les chevaux gagnants. Après chaque " saut ", l'éleveur racle chacun des coursiers avec un bâton de sueur qui lui est propre, afin de vérifier l'avancée de l'entraînement, visible dans la consistance et la couleur de la transpiration. L'éleveur sent et goûte systématiquement la sueur, qui change au cours de l'entraînement. Elle devient moins épaisse et moins salée au fur et à mesure que le cheval prend des forces, se purge et se muscle. En revanche, l'attache et l'alimentation du coursier constituent un motif poétique riche, apprécié dans les louanges des chevaux gagnants, ainsi que l'illustre cet extrait.

Voici le propriétaire du cheval

Arrivé en se dépêchant à la troisième place

Dans cette belle fête universelle

Qui réunit pour l'occasion le peuple paisible

Je chante pour attribuer ce titre

A la monture ainsi décrite :

Elle possède trois longes respectivement fixées au sol, à l'arbre et au cycle des transmigrations

Elle peut courir sur la plaine, [dans les montagnes] et les ravins

Elle a mangé du poireau sauvage, de l'oignon sauvage et des lichens

Elle est semblable à la monture précieuse, au cheval consacré et au cheval

domestique

Elle possède pour maîtres les humains, les Cieux maléfiques et les Cieux vénérés !

Tümen ölzij tegš učral büreldegsen

Tügeemel ene sajhan najryn

Gurav dugaart güjž irsen 


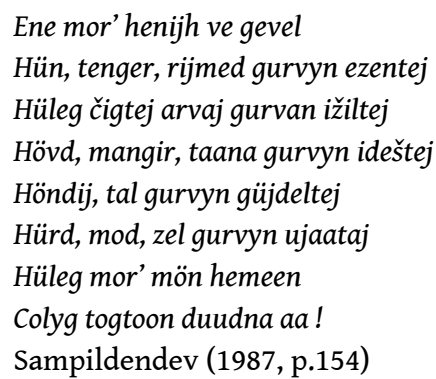

Parallèlement, les louanges du « riche estomac $»^{51}$ reconnaissent l'importance de ces savoir-faire pour obtenir la victoire dans les courses. L'une d'elles comporte les mentions suivantes : «Le maître qui l'a entraîné devenant paresseux (...) Le maître qui l'a entraîné ayant bien adapté la longe et l'attache... $»^{52}$. Cette louange, nous l'examinerons dans la conclusion, attribue la défaite aux obstacles rencontrés, à la paresse de l'entraîneur et à l'inexpérience de l'enfant. Une autre louange glorifie également le bajan hodood en ces termes: "On le dit aussi rapide que le permet son attache (...) Le maître de cette monture, faisant une erreur de jugement, l'a attachée trop court à l'ujaa... $»^{53}$. L'entraîneur de coursiers possède un savoir-faire indispensable, mais ses défaillances expliquent la défaite du « riche estomac ».

Le matin de la course, cheval et cavalier font plusieurs fois le tour de l'ujaa

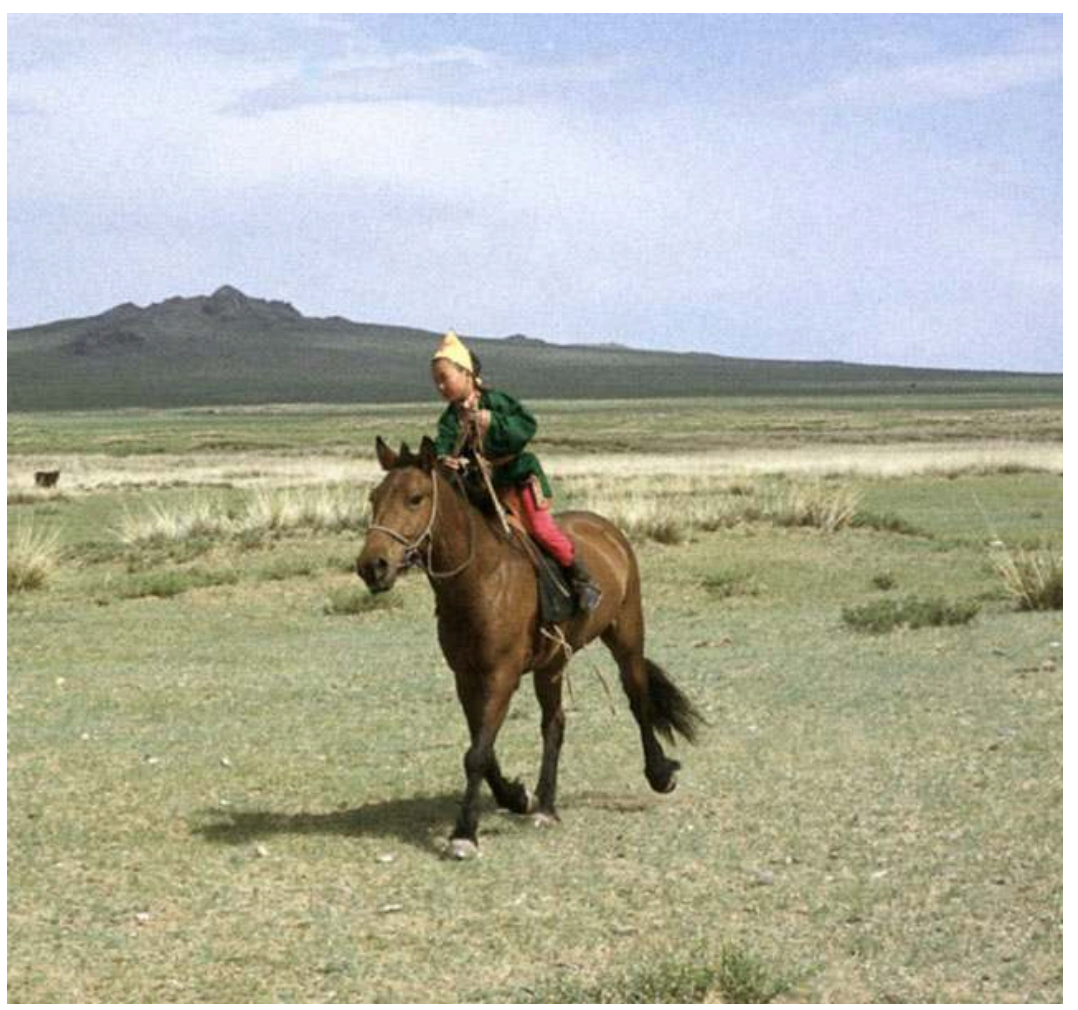

Juin 1998, Övörhangaj, Harhorin

Photographie de Gaëlle Lacaze

\section{Le cavalier et sa monture}

La préparation aux courses s'achève par le toilettage des crins du coursier et l'habillage du cavalier. Taillée régulièrement, la crinière des hongres ne réclame aucun soin 
spécifique avant la course. En revanche, celle des étalons, laissée longue, est tressée pour la course. Les crins de la queue et du toupet sont coiffés le matin même, noués à l'aide d'une ficelle rouge, et dénoués le soir. Le toupet se dresse ainsi en houppe tandis que, dans la queue, une (pour les étalons) ou deux (pour les hongres) mèches laissées libres représentent les ailes du cheval rapide. Les louanges aux chevaux utilisent régulièrement la métaphore du vol pour évoquer la rapidité des coursiers. Elles développent à foison l'assimilation du coursier à un oiseau: à l'oiseau mythique bouddhique Garuda (Han Gar'd), à un épervier (harcaga), à un aigle (bürged), etc.

41 L'enfant porte un costume spécifique pour les courses, généralement un surtout (deel), avec un dossard comportant le numéro officiel du participant. Il ne porte pas de chaussures pour être léger. La plupart des enfants monte à cru ou avec une selle rudimentaire et sans étriers. Pour stimuler sa monture, le cavalier n'utilise qu'une cravache et des psalmodies gijngoo d'intensité variable. Un mor'ny col dédié à un cheval arrivé à la neuvième place en parle ainsi :

Il s'amuse de la selle de santal,

Étonne le passant

Et se laisser monter par une jeune personne

Il est renommé parmi la foule

Jusqu'à des terroirs éloignés

Il peut encore augmenter sa vitesse

En recevant l'affection du public

Dont il attire rapidement l'attention

Son enfant [est] maigre et léger

Et son tapis de feutre fin

Il possède une morphologie rare

Et les caractéristiques du lion

Il se hâte alors qu'il tire sur son mors

Aux anneaux en argent de Hajlaar ${ }^{54}$

Il augmente le rythme de ses bonds

En entendant le son des cris $^{55}$

Zaluu hün unuuštaj

Zamyn hün gajhuuštaj

Zandan emeel tuhuuštaj

Hol gazar

Hotol olondoo aldartaj

Holboo barih hurdtaj

Nijt olny duryg tataž

Nemen hurdlah čadaltaj

Hövön nimgen tohomtoj

Höngön taaruu hü̈̈hedtej

Arslangijn šinžtej

Armagijn bajdaltaj

Hajlaar möngön zavžaaraa

Hazaar daran hurdaldag

Hašgirah čimee sonsood

Harajh erč n' nemegddeg...

(ibid., p. 161-162) 


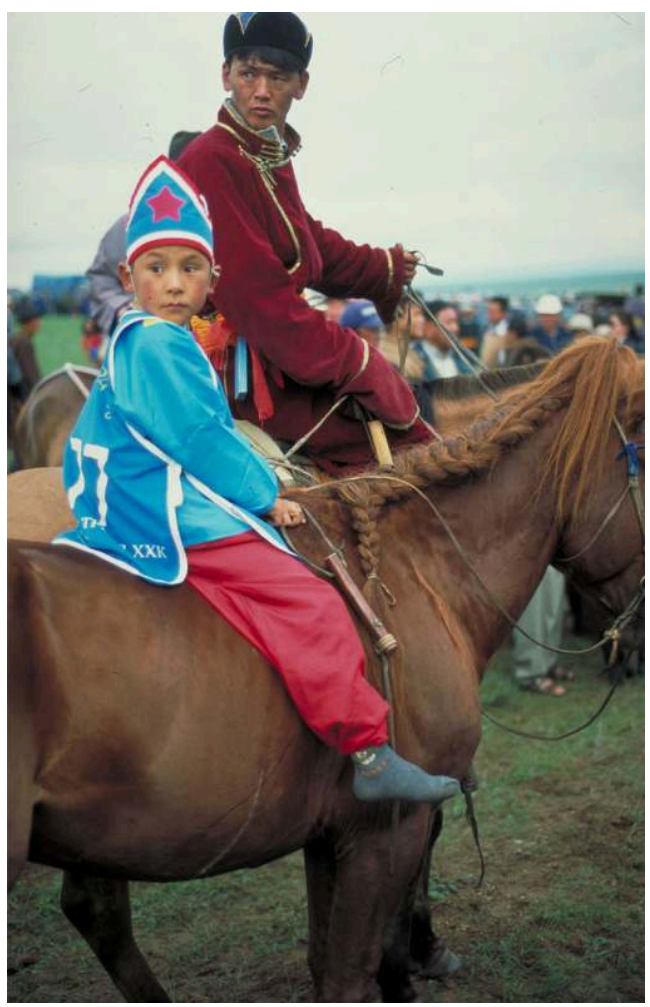

Naadam des 100000 chevaux, août 1998, Övörhangaj, Harhorin, jubilée de la capitale de l'ajmag et célébration du $700^{\mathrm{e}}$ anniversaire de la ville

Photographie de Gaëlle Lacaze

Pour conclure, nous soulignerons que la victoire dans les courses témoigne de la perfection du cheval, définie comme la conjugaison de ses qualités personnelles, du savoir-faire de l'entraîneur et de la richesse de son environnement. La victoire confirme que l'ujaačin a choisi le bon coursier et l'a correctement entraîné. Elle confirme également la préparation adéquate du cavalier et le choix du harnais approprié.

43 Les louanges adressées au perdant des perdants décrivent aussi le "riche estomac" comme un cheval parfait. À l'instar des gagnants, la vitesse de sa course est comparée au vol d'un rapace. Une louange au "riche estomac » évoque la largeur de ses épaules et de sa croupe pour insister sur la vitesse de sa course. Elle décrit ses naseaux, suggérant que son appareil respiratoire développé témoigne de sa capacité à gagner les courses. Elle lui promet, d'ailleurs, une victoire prochaine.

Parmi les gagnants de ce Naadam,

Les caractéristiques de cette monture,

Attachée sans aucune défaillance

Par son maître renommé ..., sont :

Sa course sous la pluie,

Son galop dans le soleil perçant,

Son front complètement lisse

Et son museau busqué d'aigle

Il possède les mécanismes de la vitesse du vent

A la racine des ailes du nez,

Il possède les mécanismes de la vitesse de l'eau

A la racine de la clavicule, 
Une prompte vitesse dans les muscles

Et la bonne fortune dans sa queue... ${ }^{56}$

Il possède quatre canines [semblables aux défenses] de l'éléphant

Et six vertèbres complémentaires

Il remue la poussière de la large vallée

Et réjouit la progéniture des humains.

Ene naadamd türüülehijg n'

ezen ard ... n'

ergelegüj medež ujasan

ene hülgijn šinž n'

hur boroond güjdeltej

hurc narand davhiataj

Bitüü tolimon magnajtaj

Bürged bögtör hošuutaj

Samsaanyhaa ehend

Salhind hurdny on'stoj

Omruunyhaa ehend

Usan hurdny on'stoj

Bušuu türgen n' bulčindaa

Bujan hišig n' süülendee

Zaan dörvön sojootoj

Zaamal zurgaan hüzüütej

Höndij toosyg hödölgödög

Hümüün ürijg bajasgadag.

Sampildendev (1987, p. 174)

Les louanges au « riche estomac » confirment la perfection du coursier en attribuant la défaite au défaut de préparation, aux erreurs de l'entraîneur ou à celles du cavalier. La louange précédemment citée se termine par la strophe :

Le « riche estomac » de cette année

Sera le « front de la fête",

Le « premier de la multitude » l'année prochaine,

Bien qu'il surgisse en bondissant,

Il se traîne comme un char à bœufs

Il a fait différents écarts

Il s'est cogné dans de larges buttes

Il a sans cesse rencontré divers obstacles

Il a trébuché dans de larges trous de souris

Il est monté par un enfant inexpérimenté

Tenant une cravache trop petite!

Unasan hü̈̈hed n' balčirdaad

Bar'san tašuur n' boginidiid

Ülij manhan dajraldaad

Ürgelž saatal tohioldood

Ereg manhan dajraldaad

Eldev haduural tohioldood

Üserč garsan bolovč

Üher tereg šig hocorč

Ene žilijn bajan hodood

Ireh žilijn bajaryn magnaj

Bat tümnij eh ee!

(ibid., p. 174-175)

Dans le Naadam, le coursier, même perdant, reste donc parfait. On loue le perdant des perdants, le « riche estomac » en des termes comparables à ceux destinés aux gagnants, voire avec des qualificatifs propres au(x) gagnant(s) des gagnants, le(s) premier(s) de la 
course des étalons. Une louange au neuvième cheval (Jös düügeer mor'ny col) le décrit ainsi :

... Quand il s'agit de décrire

La parfaite morphologie et

Les signes virils

Du cheval pie

Éblouissant comme l'éclair,

Bruyant comme la forge

Et la grêle,

Sonore comme la grêle

Et [le vol] du faucon

Qui se rapproche

Du lieu de la capture...

... Bariany gazar

Ojrton irehed

Duut möndör

Dujlan harcaga

Širï̈n möndör

Širmen duut

Ajanga met gjalalzan

Irdeg ene morind

Er hojor zagalyn šinž

Tögöldör büreldsenijg

Tösöölž helehed...

(ibid., pp. 162-163)

Les louanges qui promettent au "riche estomac " une victoire à venir produisent un discours performatif qui abolit la distinction entre le gagnant et le perdant. Dans l'idéologie des courses de chevaux, chacun peut donc gagner, la chance tourne. Cette conception respecte l'idéologie générale du naadam. Elle renvoie à une représentation circulaire du temps, chaque course réactualisant l'aléatoire de la "chance » (hijmor'). Cette notion prend ici un sens particulier car, dans les éloges examinés, les chevaux doivent leur victoire à la grâce obtenue des divinités bouddhiques. Ainsi, divers aspects de leur organisation suggèrent que les courses peuvent offrir un support d'expression à différentes conceptions du bouddhisme ${ }^{57}$. En outre, elles servent les enjeux et glorifient l'image de l'institution officielle promotrice du Naadam. 


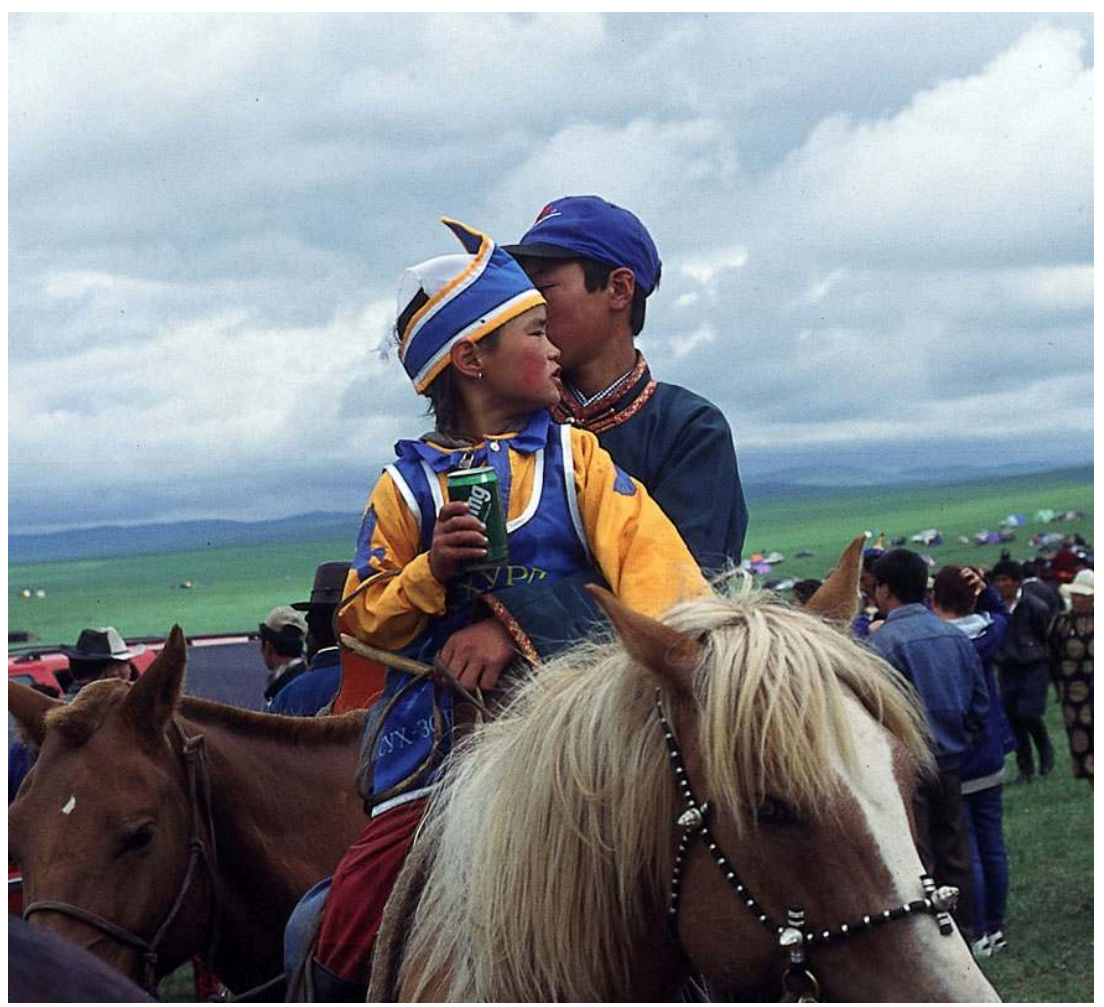

Naadam des 100000 chevaux, août 1998, Övörhangaj, Harhorin, jubilée de la capitale de l'ajmag et célébration du $700^{\circ}$ anniversaire de la ville

Photographie de Gaëlle Lacaze

Premièrement, les naadam ont été profondément modifiés à la suite de leur édification en rituel durant la domination mandchoue. Deuxièmement, la première mention de courses de chevaux durant le Naadam apparait après l'allégeance des Halh à la dynastie Qing. Et troisièmement, le principe qui distingue les gagnants, le hijmor', est un aspect de la «chance " spécifique au champ lexical bouddhique. Il est donc probable que les courses de chevaux aient été introduites dans les festivités du Naadam, érigé en rituel politique et religieux au cours du xvII ${ }^{e}$ siècle. Ainsi, la dynastie Qing aurait souhaité promouvoir ce jeu populaire afin de l'utiliser comme alternative à la lutte. En outre, renouvelant la victoire chaque année et la garantissant d'une année sur l'autre, les courses de chevaux offrent à l'idéal social un support privilégié d'expression de notions communautaires. Elles n'ont donc pas subi trop de pression pour être adaptées ou éradiquées durant la période socialiste, à la différence de la lutte ou du tir à l'arc.

Depuis 1990, les courses de chevaux supportent de nouveaux objectifs et revêtent de nouveaux enjeux, d'abord économiques. En effet, la propriété des chevaux de course permet de constituer un capital réel et potentiel, constitué par les étalons de course souvent destinés à engendrer d'autres gagnants. Les premières écuries professionnelles apparaissent entre les mains des "nouveaux [riches] Mongols ». L'entraînement devient, quant à lui, un savoir-faire facilement monnayable et économiquement très intéressant. $\mathrm{Si}$, avant la période socialiste, les riches éleveurs qui ignoraient les règles d'entraînement des coursiers ou qui voulaient augmenter leurs chances de gagner rémunéraient des dresseurs spécialisés, la nouveauté réside dans la grande rentabilité de cette activité. 
En outre, les courses suscitent l'enthousiasme de la population locale, sensible au prestige que rapportent les chevaux vainqueurs, entraînés par des ujaačin renommés et possédés par les Mongols les plus riches. L'idéal égalitaire et communautaire des courses est aussi revendiqué comme un aspect attractif, le personnage du « riche estomac » soulevant l'affection de l'ensemble des spectateurs. La propension à garantir la circulation de la chance et à permettre l'adhésion populaire aux idéaux nationaux du naadam assure aux courses de chevaux l'engouement du public mongol, des citadins comme des ruraux, des hommes comme des femmes. Et la place qu'occupent aujourd'hui les courses dans le modèle identitaire de la République de Mongolie et dans l'idéologie du Naadam se trouve renforcée par le flux touristique de cavaliers internationaux qui y assistent en masse à travers le pays.

\section{BIBLIOGRAPHIE}

Aubin, F.

1999 Critères d'appréciation des chevaux dans la tradition des nomades mongols, Cahiers de la société des Études euro-asiatiques, 8. Le Cheval en Eurasie, pratiques quotidiennes et déploiements mythologiques (Paris, L'Harmattan), pp. 65-86.

1986 L'art du cheval en Mongolie, Production pastorale et Société, 19, pp.129-149.

Caillois, R.

[1958] 1991 Des jeux et des hommes (Paris, Éditions Gallimard, Folio Essais).

Ferret, C.

2004 De l'attache des chevaux à la fécondation des femmes en passant par la cuisine. Quelques pistes pour l'exploration des notions altaïques de chaud et de froid, Études rurales, 171-172, pp. 243-270.

Gaadamba, S. et D. Cerensodnom

1978 Mongol ardyn aman zohiolyn deež bičig [Le meilleur de la littérature orale populaire mongole] (Ulaanbaatar, Ulsyn hevlelijn gazar).

Galindiv, R.

1977 Erijn gurvan naadam [Les trois jeux virils], (Ulaanbaatar, Ulsyn Hevlelijn gazar).

Even, M.-D. et Pop, R. [trad.]

1994 Histoire secrète des Mongols (Mongghol-un ni'uca tobciyan) Chronique mongole du XIIIe siècle, Traduit et annoté par Even et Pop, (Paris, Gallimard).

Kabzinska-Stawarz, I.

1991 Games of Mongolian shepherds (Warszawa, Institute of the history of material culture, Polish Academy of sciences).

Lacaze, G.

2000 Rite de renouveau ou fête nationale : la lutte et le tir à l'arc dans les jeux virils mongols, Études Mongoles et Sibériennes, 30-31, pp. 73-123.

2005 Obraz Čingis hana v konstruirovanii identičnosti mongol'skih narodov postsocialističeskogo perioda, Ab imperio, 4, pp. 113-134. 
2006 Games of power: State Control, the Naadam and the Cult of Chinggis Khan, in D. Sneath (ed.) States of mind: Power, place and the subject in Inner Asia (Washington, Cambridge, CEAS, MIASU), pp. 91-108.

Magsaržav, $\mathrm{H}$.

1960 Doloon hušuu naadam [Le Naadam des Sept Bannières], Etnografijn sudlal I, fasc.7

Mönh, C.

1964 Ardyn jaruu najrgijn tuhaj [À propos de la poésie populaire], (Ulaanbaatar, Ulsyn hevlelijn gazar).

Šagdargočoo, C.

1960 Erijn gurvan naadmyn jaruu najrag [Poésie populaire des Trois jeux virils], (Ulaanbaatar, Ulsyn Hevlelijn gazar).

Sampildendev, $\mathrm{H}$.

1987 Mongol ardyn zan üjlijn aman zohiol [Littérature orale sur les coutumes populaires mongoles], (Ulaanbaatar, Ulsyn Hevlelijn gazar).

Pokropek, W., M. Turnau \& A. Wozniak

1991 Mongol's games (Warszawa, Institut of the History of Material Culture, Polish Academy of Sciences).

Žukovskaja, N. L.

1989 Godovoj cikl : Mongoly, in Kalendarnie obyčaj i obrjady narodov Vostočnoj Azij [Habitudes et cérémonies calendaires chez les populations est-asiatiques. Cycle annuel : la Mongolie] (Moskva, NAUKA, Glavnaja vostočnoj literatury), pp. 233-263.

Zorig, R.

1960 Mongol ardyn sport [Les sports populaires mongols] (Ulaanbaatar, Ulsyn hevlelijn gazar).

\section{NOTES}

1. J'emploie Naadam avec une majuscule pour désigner les festivités durant lesquelles on joue aux naadam « jeux » (lutte, courses de chevaux et tir à l'arc), qu'elles soient nationales ou locales. La notion de « compétition virile » et chacun des jeux seront désignés par le terme naadam, avec une minuscule.

2. Traditionnellement, les lutteurs étaient des hommes célibataires qui s'affrontaient en vue de leur mariage. A la campagne, dans certains petits Naadam, ils restent de jeunes célibataires.

3. Les données fournies proviennent de recherches effectuées en Mongolie: lors du Naadam d'Ulaanbaatar, dans le cadre de ma maîtrise d'ethnologie (soutenue en 1993 à Paris X-Nanterre) et d'autres, réalisées ultérieurement jusqu'en 2009, en particulier, en 2003-2004, dans le cadre d'une collaboration CEMS (EPHE-Paris) - MIASU (King's College - Cambridge University, UK).

4. Dans la lutte et le tir à l'arc, l'alea « la chance » que le joueur doit posséder s'exprime par le terme az (voire az žargal) tandis que, dans les courses, le couple formé par le cavalier et sa monture doit bénéficier de hijmor', un terme qui renvoie en mongol à l'idée de "grâce ", de «fortune », accordée par une divinité transcendante. La chance indispensable aux courses est ainsi liée à la notion de hijmor' "cheval de vent", particulièrement importante dans le bouddhisme mongol.

5. La comparaison des illustres épopées de Žangar et Geser renforce l'hypothèse d'une introduction tardive des courses de chevaux dans le Naadam, liée au processus de conversion des Mongols au bouddhisme. En effet, les Naadam mentionnés dans l'épopée mongole de Žangar (répandue chez les Mongols septentrionaux) ne comportent pas de course de chevaux, tandis que 
ceux décrits dans l'épopée d'origine tibétaine de Geser (davantage connue chez les Mongols méridionaux) en comportent systématiquement.

6. Ainsi, dans l'Histoire secrète des Mongols (Even et Pop 1994, § 140, § 245), il n'est jamais fait mention de courses de chevaux dans le Naadam (concernant les utilisations idéologiques du Naadam, voir Lacaze 2006). La première mention des courses de chevaux dans une festivité officielle concernerait les Doloon hušuu naadam " Naadam des Sept bannières ", organisés après 1691, date de l'allégeance des Halh à la dynastie Qing (Magsaržav 1960).

7. Selon R. Zorig (1960, pp. 12-15), le Naadam n'acquiert de signification rituelle qu'avec la domination mandchoue et la pénétration du bouddhisme. Il passe alors du statut de démonstration militaire, organisée à l'échelle des armées de provinces ou entre celles-ci, à celui de cérémonie célébrée au pied des montagnes sacrées lors des sacrifices tri-annuels consacrant le représentant de l'Empereur mandchou.

8. Au Tibet, des courses de chevaux sont présentes dans les jeux organisés pour les rituels aux cairns (latse), qui pourraient avoir exercé une influence sur les Mongols.

9. Jusqu'en 2003, l'arrivée des courses avait lieu sur l'aire de Jarmag, au S.-O. d'Ulaanbaatar, sur la route de l'aéroport Bujant-Uhaj / Činggis Qan. En raison de l'extension de la ville, les courses ont été délocalisées au lieu-dit Huj Doloon hutagt, à quarante kilomètres au N.-E. de la capitale.

10. La date du Nouvel an, durant le Mois blanc, se calcule selon un calendrier luni-solaire et change tous les ans. Elle se situe généralement entre la fin du mois de janvier et celle du mois de février.

11. Notons que, depuis 2006, avec l'essor du tourisme, les provinces méridionales de Mongolie organisent des Naadam d'hiver qui comportent également des courses de chameaux.

12. Nous verrons ultérieurement le rôle emblématique que joue cet étendard dans les louanges aux chevaux.

13. Les Mongols ignorent le sens de ce mot, psalmodié pour donner du courage au coursier. Selon certains de mes informateurs, gijngoo serait un mot tibétain signifiant « chance ».

14. Traditionnellement, seuls les garçons de six à douze ans montaient les chevaux de course. Si des petites filles jouent parfois aujourd'hui le rôle de cavalier, à défaut de garçons, en République de Mongolie, c'est rarement le cas dans les régions mongoles de Chine, en République Autonome de Mongolie-Intérieure (RAMI), où les chevaux des courses sont parfois montés par des hommes adultes.

15. Autrefois improvisées, les louanges chantées pour glorifier les gagnants des courses constituent un genre prolifique de la poésie mongole. Les louangeurs (collogč) improvisaient à loisir autour des thèmes stéréotypés et répandus dans ce genre littéraire, se distinguant par là les uns des autres. Actuellement, certains thèmes et formules semblent fixés dans la mémoire des louangeurs et se retrouvent même dans des chansons de variété ou des morceaux de pop-folk (voir, entre autres, les chansons consacrant un idéal de vie rural et qui font l'éloge des troupeaux de chevaux mongols). Nous examinerons ici plusieurs strophes de diverses louanges, disponibles dans des ouvrages sur le folklore et principalement issues des ouvrages suivants: Sampildendev 1987, Gaadamba \& Cerensodnom 1978 et Šagdargočoo 1960.

16. Ce titre - nous y reviendrons - qualifie le grand perdant des courses, c'est-à-dire, le perdant de la course la plus courte, celle des plus jeunes coursiers.

17. L'« attrapeur de chevaux" monte un hongre dressé pour la capture à l'uurga (perche à l'extrémité de laquelle est fixée une boucle), c'est-à-dire un cheval entrainé à évoluer au sein de la manade et pouvant être conduit avec la seule assiette, ce qui permet de libérer les mains, même au galop.

18. Les épithètes constituent un genre particulier de la poésie orale liée au Naadam. Elles glorifient les gagnants à répétition de chacun des trois naadam, dont les victoires se succèdent d'une année sur l'autre. Appelées čimeg, littéralement «bijou, ornement, décoration ", elles seraient plus tardives que les louanges elles-mêmes. En effet, leur contenu métaphorique reste 
très marqué par une idéologie étatique et nationaliste. On retrouve des épithètes comparables dans les titres des lutteurs ou des tireurs à l'arc. Ces épithètes sont compilées, entre autres, dans Sampildendev 1987, p. 172-173 et Šagdargočoo 1960, p. 45-52.

19. Nisen güjcež nemen hurdaldag hüleg (Sampildendev 1987 p. 172, pour cette expression et les suivantes).

20. Žims hangajn žargalt hüleg.

21. Hujd güjcehgüj hurdan hüleg.

22. Hariltgüj hurdan handgajn hüleg.

23. Agtyn türüünd güjdeg armag hüleg.

24. Uls törijn ulaan suunag.

25. Sajn törijn saaršgüj hüleg.

26. Ezen törijn itgelt hüleg.

27. Bat törijn bahdalt hüleg.

28. Huv'sgalt törijn hurdan hüleg, geh met.

29. Deux versions proches de cette louange sont consultables dans Mönh 1964, pp. 81-83 et Sampildendev 1987, pp. 173-175.

30. Par exemple, la victoire des lutteurs est attribuée, entre autres, à la richesse des pâturages de leur région d'origine, car celle-ci a permis de produire la viande et le lait de jument fermenté qui forgent la force des gagnants.

31. «Türüülsen mor'ny col» (Sampildendev 1987, pp. 165-166).

32. Büren erht BNMAU-yn ... ajmgijn ... sum negdelijn ... dugaar sürgijn udamt azargany ür !

33. Le Cam est un rituel effectué par des moines bouddhistes, masqués et costumés. Ces danses, accompagnées de différentes pratiques liturgiques, représentent la victoire des divinités du bien sur celles du mal et possèdent une fonction propitiatoire pour l'ensemble de la communauté des croyants.

34. Deux versions proches de cette louange sont consultables dans Sampildendev 1987, pp. 153-155 et dans Gaadamba et Cerensodnom 1978, pp. 132-133.

35. Le mont Sumeru est sacré dans le bouddhisme.

36. Sampildendev ibid., p. 173.

37. Ce mor'ny col est également cité par C. Šagdargočoo(1960, pp. 31-37).

38. «Bajan hodoodny col » (Sampildendev 1987, p. 175).

39. Les Halh constitue la population majoritaire en Mongolie, où le mongol halh est la langue dominante.

40. Uraldahaar ulaan tugijn hamt; Ulsyn olon mor'dtoj garč bajhdaa ; Bajartaj č jum šig sanagda bajlaa.

41. Pour celui qui arrive à la huitième position : eznee bajasgagč, eremgij hüleg. Pour le neuvième : Tümnij bajasgalan, tögs hurdan hüleg (« Mor’ny čimeg » - Sampildendev 1987, pp. 172-173).

42. Ujaa désigne la longe d'attache suspendue entre deux piquets, à près de deux mètres audessus du sol. Et le verbe ujaa- prend le sens de "entraîner pour les courses ». L'entraîneur (ujaačin) est l'agent lié à cette activité et à cette longe. Sur le rôle central de l'attache dans l'entraînement, voir Ferret 2004 ainsi que, dans le présent recueil, l'article de C. Ferret et A. Toktabaev.

43. Les hurdan mor'ny šinž "caractéristiques du coursier " constituent un riche répertoire spécifique aux éleveurs de chevaux en général, et aux entraîneurs de coursiers en particulier (cf. Aubin 1999, pp. 65-86).

44. La version principale de cette «Louange au cheval gagnant» est mentionnée par H. Sampildendev dans Mongol ardyn zan üjlijn aman zohiol (1987, p.165-166) et la version comportant également la rime soulignée est mentionnée par C. Šagargočoo dans Erijn gurvan naadam jaruu najrag (1961, p. 36-37).

45. Les « sauts » (üserge) sont des petites courses d'entraînement (cf. infra). 
46. Littéralement [couleur de] borax - blanc-grisâtre, jaunâtre, verdâtre. Le borax est tunsaa en mongol, et non tunsag comme noté dans le texte.

47. La rime soulignée provient d'une autre version citée par S. Gaadamba et D. Cerensodnom (1978, p. 132).

48. Tout rassemblement constitue un événement pour les Mongols de tradition pastorale nomade. Rares sont ceux qui ne prennent pas une allure festive. Les entraînements des coursiers offrent l'occasion aux éleveurs d'une même région de se réunir après un long hiver. Ils constituent toujours une festivité souvent informelle et presque improvisée. Certains peuvent même servir de prétexte à la célébration d'un événement local ou collectif (par exemple, la fête du canton ou tout simplement d'un lieu dit).

49. Sur les détails de l'entraînement kazakh des chevaux de course, qui comporte bien des points communs avec le mongol, voir l'article de C. Ferret et A. Toqtabaev dans le présent recueil (note de l'éditeur).

50. Pour davantage de détails concernant cette procédure, voir la thèse de Laetitia Merli, De l'ombre à la lumière, de l'individu à la nation: Renouveau du chamanisme en Mongolie postcommuniste, dirigée par Philippe Descola (2004, EHESS Paris).

51. «Bajan hodoodny col » (Sampildendev 1987, p. 175).

52. Ujasan ezen n' zalhuudaž; [...] Ujasan ezen n' ujaa sojlgyg n' taaruulž...

53. Ujaa n' tohirood hurdan gedeg; [...] ene hülgijn ezen n'; endüürč tašaarč bodsonoos; Ujasan ujaa n' bagadaad... (Ibid., p. 174).

54. Dans le district de Hailar, situé dans une province orientale de RAMI, vivent majoritairement, des Chinois ainsi que des Mongols Barga et des Evenks.

55. Cette louange est également citée par C. Šagdargočoo, 1960, p. 32-33.

56. Cette louange est également citée par Mönh, op. cit. 1964, pp. 81-83.

57. Elles restent, par exemple, idéologiquement compatibles avec les représentations psychopompes que le bouddhisme mongol attribue au cheval.

\section{RÉSUMÉS}

En Mongolie, les courses de chevaux sont le plus récent, mais néanmoins le plus apprécié des naadam « jeux ». Elles remportent l'adhésion du public mongol et international. Leur examen, à travers les pratiques qu'elles impliquent et la littérature orale à laquelle elles donnent naissance, offre une lecture des éléments constitutifs de ce naadam et de l'idéologie qu'il véhicule. Les courses de chevaux nécessitent une préparation précise et sollicitent des savoir-faire qui font la fierté des éleveurs mongols. Le rôle du « riche estomac ", le "perdant des perdants ", révèle une conception de la chance circulaire et re-distributive. Il met en évidence le rôle politique des courses qui supportent une fonction propitiatoire, promettant le renouvellement et une redistribution plus égalitaire de la « chance ».

In Mongolia, the horse races are the most recent, but nevertheless the most appreciated of the naadam games. They have gained the full support of Mongolian and international audiences. Studying them through the practices related to them and the oral literature they have given rise to allows us to piece together the elements that make up this naadam and the ideology behind it. The horse races require meticulous preparation and know-how of which Mongolian breeders are very proud. The role of the "rich stomach" (the loser among losers) reveals a conception of 
circular and redistributive luck. It underlines the political role of the races, which have a propitiatory function, promising the renewal of and a more egalitarian redistribution of the "good fortune". The Mongolian words used for it, like benediction or grace, have a Buddhist connotation.

\section{INDEX}

Mots-clés : naadam, rituel de renouveau, course de chevaux, entraînement, littérature orale, victoire, chance

Thèmes : rituel, animal, élevage, fête, identité, idéologie, institution Index géographique : Mongolie

Keywords : naadam, ritual of renewal, horse races, training, oral literature, victory, good fortune nomsmotscles Mongol

\section{AUTEUR}

\section{GAËLLE LACAZE}

Gaëlle Lacaze est maître de conférence à l'Université de Strasbourg. Spécialiste des peuples mongols, elle fait des recherches en anthropologie du corps, du nomadisme et de l'espace et utilise de nombreuses techniques de l'anthropologie visuelle. 